(C) 2018 Elsevier. This manuscript version is made available under the CC-BY-NC-ND 4.0 license http://creativecommons.org/licenses/by-nc-nd/4.0/

\title{
Quantum and non-signalling graph isomorphisms
}

\author{
Albert Atserias ${ }^{1}$, Laura Mančinska ${ }^{2}$, David E. Roberson ${ }^{3}$, Robert Š́mal ${ }^{4}$, Simone \\ Severini $^{5}$, and Antonios Varvitsiotis ${ }^{6}$ \\ ${ }^{1}$ Universitat Politècnica de Catalunya, Spain \\ ${ }^{2}$ University of Bristol, United Kingdom \\ ${ }^{3}$ University College London, United Kingdom \\ ${ }^{4}$ Charles University, Czech Republic \\ ${ }^{5}$ University College London, United Kingdom and Shanghai Jiao-Tong University, China \\ ${ }^{6}$ Nanyang Technological University and Centre for Quantum Technologies, Singapore
}

October 25, 2018

\begin{abstract}
We introduce a new two-player nonlocal game, called the $(G, H)$-isomorphism game, where classical players can win with certainty if and only if the graphs $G$ and $H$ are isomorphic. We then define the notions of quantum and non-signalling isomorphism, by considering perfect quantum and non-signalling strategies for the $(G, H)$-isomorphism game, respectively. First, we prove that non-signalling isomorphism coincides with the well-studied notion of fractional isomorphism, thus giving the latter an operational interpretation. In the quantum case, we consider both the tensor product and commuting frameworks for nonlocal games. Second, we show that, in the tensor product framework, quantum isomorphism is equivalent to the feasibility of two polynomial systems in non-commuting variables, obtained by relaxing the standard integer programming formulations for graph isomorphism to Hermitian variables. Finally, we provide a construction for reducing linear binary constraint system games to isomorphism games. This allows us to determine quantum isomorphic graphs that are not isomorphic. Furthermore, it allows us to show that our two notions of quantum isomorphism, from the tensor product and commuting frameworks, are in fact distinct relations, and that the latter is undecidable. Our proof techniques are related to the Feige, Goldwasser, Lovász, Safra, and Szegedy reduction from the inapproximability literature [J. ACM, 43(2):268-292, 1996], as well as the construction due to Cai, Fürer, and Immerman [Combinatorica, 12(4):389-410, 1992].
\end{abstract}

\section{Introduction}

Given graphs $G$ and $H$, an isomorphism from $G$ to $H$ is a bijection $\varphi: V(G) \rightarrow V(H)$ such that for all $g, g^{\prime} \in V(G), \varphi(g)$ is adjacent to $\varphi\left(g^{\prime}\right)$ if and only if $g$ is adjacent to $g^{\prime}$. When such an isomorphism exists, we say that $G$ and $H$ are isomorphic and write $G \cong H$. The graph isomorphism (GI) problem consists of deciding whether two graphs are isomorphic. It is a question with fundamental practical interest due to the number of problems that can be reduced to it. Additionally, the GI problem has a central role in theoretical computer science as it is one of 
the few naturally defined problems in NP which is not known to be polynomial-time solvable or NP-complete. While there is a deterministic quasipolynomial algorithm for the GI problem [5], regardless of its worst case behavior, it can be solved with reasonable efficiency in practice (e.g. see [19]). In relation to the context of this paper, an important line of research concerning graph isomorphism focuses on the analysis of many equivalence relations that form hierarchical structures. Prominent instances are, for example, cospectrality, fractional isomorphism, and the Sherali-Adams hierarchy of relaxations, e.g. see [3, 4, 13, 35].

Integer programming formulations. As is the case for all integer constraint satisfaction problems, the GI problem can be formulated as an integer programming problem. Our next goal is to give two of these formulations as they are relevant to this work. The first one is an integer quadratic program and the second one an integer linear program. We note that several recent developments concerning the GI problem are based on hierarchies of linear programming relaxations of its integer linear programming formulation we give below (e.g. see [3, 14]).

Consider two graphs $G$ and $H$ with adjacency matrices $A_{G}$ and $A_{H}$ respectively. Recall that the adjacency matrix, $A_{G}$, of a graph $G$ is a symmetric matrix whose rows and columns are indexed by $V(G)$, and such that $A_{g g^{\prime}}=1$ if $g$ is adjacent to $g^{\prime}$, and $A_{g g^{\prime}}=0$, otherwise. Throughout this work we will only consider undirected loopless simple graphs. In the formulation below, and throughout this work, we will use $\operatorname{rel}\left(g, g^{\prime}\right)$ to denote the relationship of $g$ and $g^{\prime}$, i.e., $\operatorname{rel}\left(g, g^{\prime}\right) \in\{=, \sim, \not\}$ depending on whether $g$ and $g^{\prime}$ are equal, adjacent, or distinct and non-adjacent. It is easy to verify that $G \cong H$ if and only if the integer quadratic program

$$
\begin{aligned}
x_{g h}^{2} & =x_{g h}, \text { for all } g \in V(G), h \in V(H) ; \\
\sum_{h^{\prime} \in V(H)} x_{g h^{\prime}} & =\sum_{g^{\prime} \in V(G)} x_{g^{\prime} h}=1, \text { for all } g \in V(G), h \in V(H) ; \\
x_{g h} x_{g^{\prime} h^{\prime}} & =0, \text { if } \operatorname{rel}\left(g, g^{\prime}\right) \neq \operatorname{rel}\left(h, h^{\prime}\right),
\end{aligned}
$$

in the real scalar variables $x_{g h}$ for all $g \in V(G), h \in V(H)$ is feasible.

The second integer programming formulation for the GI problem is based on permutation matrices, i.e., square $(0,1)$-matrices with a single 1 in every row and column. Again, it is straightforward to verify that $G \cong H$ if and only if there exists an $n \times n$ permutation matrix $P=\left(p_{i j}\right)$ such that $P^{\top} A_{G} P=A_{H}$, or equivalently when the following integer linear program is feasible:

$$
\begin{aligned}
p_{i j}^{2} & =p_{i j}, \text { for all } i, j \in[n] ; \\
\sum_{\ell=1}^{n} p_{\ell j} & =\sum_{k=1}^{n} p_{i k}=1, \text { for all } i, j \in[n] ; \\
A_{G} P & =P A_{H}, \text { where } P=\left(p_{i j}\right) .
\end{aligned}
$$

By the Birkhoff-von Neumann theorem, the convex hull of the set of $n \times n$ permutation matrices is equal to the set of $n \times n$ doubly stochastic matrices, i.e., entrywise nonnegative matrices where the sum of the entries in each row and column is equal to 1 . This naturally suggests the following linear relaxation of the GI problem. We say that $G$ and $H$ are fractionally isomorphic, and write $G \cong_{f} H$, if there exists a doubly stochastic matrix $D$ such that $A_{G} D=D A_{H}$. This defines an equivalence relation on graphs that has been studied in detail and characterized in multiple ways [28, 33]. 
Matrix relaxations. In this work we focus on two natural matrix relaxations of $(\mathrm{IQP}$ and (ILP). First, we consider (IQP), where we relax the scalar variables $x_{g h}$ to be $d \times d$ Hermitian indeterminates $X_{g h}$. This leads to the following quadratic polynomial system in Hermitian variables:

$$
\begin{aligned}
X_{g h}^{2} & =X_{g h}, \text { for all } g \in V(G), h \in V(H) \\
\sum_{h^{\prime} \in V(H)} X_{g h^{\prime}} & =\sum_{g^{\prime} \in V(G)} X_{g^{\prime} h}=I_{d}, \text { for all } g \in V(G), h \in V(H) \\
X_{g h} X_{g^{\prime} h^{\prime}} & =0, \text { if } \operatorname{rel}\left(g, g^{\prime}\right) \neq \operatorname{rel}\left(h, h^{\prime}\right) .
\end{aligned}
$$

Note that by definition, every family of matrices $\left\{X_{g h}\right\}_{g, h}$ which is feasible for (IQP $)$ satisfies $X_{g h}^{2}=X_{g h}=X_{g h}^{\dagger}$ and thus each matrix is an orthogonal projector.

For the matrix relaxation of (ILP), we replace the permutation matrix $P$ with a block matrix $\mathcal{P}=\left[\left[P_{i j}\right]\right]$ where each block $P_{i j}$ is a $d \times d$ orthogonal projector. Thus we consider the following program in Hermitian $d \times d$ indeterminates $P_{i j}$ :

$$
\begin{aligned}
P_{i j}^{2} & =P_{i j}, \text { for all } i, j \in[n] ; \\
\sum_{\ell=1}^{n} P_{\ell j} & =\sum_{k=1}^{n} P_{i k}=I_{d}, \text { for all } i, j \in[n] ; \\
\left(A_{G} \otimes I_{d}\right) \mathcal{P} & =\mathcal{P}\left(A_{H} \otimes I_{d}\right), \text { where } \mathcal{P}=\left[\left[P_{i j}\right]\right] .
\end{aligned}
$$

Note that for $d=1$ the matrix $\mathcal{P}$ is exactly a permutation matrix.

As we will see in Section 5, the system $\left(\overline{\mathrm{IQP}_{d}}\right)$ is feasible if and only if $\left(\mathrm{ILP}_{d}\right)$ is feasible. In view of this, the feasibility of $\left(\mathrm{IQP}_{d}\right)$ (or equivalently $\left(\overline{\mathrm{ILP}_{d}}\right)$ ) corresponds to a natural relaxation of the notion of graph isomorphism, which we call quantum isomorphism (see Definition 1.1).

Although the term "quantum isomorphism" might seem unmotivated at this point, as we will see in the next section, feasibility of $\left(\mathrm{IQP}_{d}\right)$ corresponds to a relaxation of graph isomorphism based on the existence of winning quantum strategies for a certain type of game. The relaxation makes use of the mathematical formalism of quantum theory and its definition requires physical resources available in quantum mechanics (see Theorem 2).

\section{$1.1 \quad$ Nonlocal games}

A two-party nonlocal game includes a verifier and two players, Alice and Bob, that devise a cooperative strategy. The game is defined in terms of finite input sets $X_{A}, X_{B}$ and finite output sets $Y_{A}, Y_{B}$ for Alice and Bob respectively, a Boolean predicate $V: X_{A} \times X_{B} \times Y_{A} \times Y_{B} \rightarrow\{0,1\}$, and a distribution $\pi$ on $X_{A} \times X_{B}$.

In the game, the verifier samples a pair $\left(x_{A}, x_{B}\right) \in X_{A} \times X_{B}$ using the distribution $\pi$ and sends $x_{A} \in X_{A}$ to Alice and $x_{B} \in X_{B}$ to Bob. The players independently respond with $y_{A} \in Y_{A}$ and $y_{B} \in Y_{B}$, respectively. We say the players win the game if $V\left(x_{A}, x_{B}, y_{A}, y_{B}\right)=1$.

The goal of Alice and Bob is to maximize their winning probability. In the setting of nonlocal games, the players are allowed to agree on a strategy beforehand, but they cannot communicate after they receive their questions. The parties only play one round of this game, and we are concerned with strategies that win with certainty, i.e., probability equal to 1 . We refer to such 
a strategy as a winning or perfect strategy. Lastly, we will assume that the distribution $\pi$ has full support, meaning that any possible pair of inputs occurs with nonzero probability. Since we are only interested in perfect strategies, it does not matter which full support distribution $\pi$ we consider, and this allows us to characterize winning strategies purely in terms of the predicate $V$ (e.g. see (5)).

Strategies for nonlocal games. A deterministic classical strategy for a nonlocal game is one in which Alice's response is determined by her input, and similarly for Bob. In a general classical strategy, the players may also use local and shared randomness to determine their responses.

In Section 3.2 we focus on another family of strategies, where the players are allowed to use quantum resources to determine their answers. Specifically, a quantum strategy allows the players to determine their answers by performing joint measurements on a shared quantum state. It is known that such strategies can allow the players to correlate their answers beyond classically reachable limits.

In Section 3.3, we consider strategies satisfying the non-signalling constraints (see Equation (9)). Intuitively, the non-signalling property says that Alice's local marginal distributions are independent of Bob's input and, symmetrically, Bob's local marginal distributions are independent of Alice's input. Thus, Alice cannot obtain any information about Bob's input based on her input and output, and vice versa. This is the most general class of strategies we consider in this work.

For any of the above classes of strategies, the goal of the players is to determine the maximum (or supremum) probability of winning a given nonlocal game, which is known as the classical/quantum/nonsignalling value of the game respectively.

The $(G, H)$-isomorphism game. Given two graphs $G$ and $H$, we introduce a new nonlocal game which we call the $(G, H)$-isomorphism game, with the intent of capturing and extending the notion of graph isomorphism. The $(G, H)$-isomorphism game is played as follows: The verifier selects uniformly at random a pair of vertices $x_{A}, x_{B} \in V(G) \cup V(H)$ and sends $x_{A}$ to Alice and $x_{B}$ to Bob respectively. The players respond with vertices $y_{A}, y_{B} \in V(G) \cup V(H)$. Throughout, we assume that $V(G)$ and $V(H)$ are disjoint so that players know which graph their vertex is from.

The first winning condition is that each player must respond with a vertex from the graph that the vertex they received was not from. In other words we require that:

$$
x_{A} \in V(G) \Leftrightarrow y_{A} \in V(H) \text { and } x_{B} \in V(G) \Leftrightarrow y_{B} \in V(H) .
$$

If condition (1) is not met, the players lose. Assuming (1) holds we define $g_{A}$ to be the unique vertex of $G$ among $x_{A}$ and $y_{A}$, and we define $g_{B}, h_{A}$, and $h_{B}$ similarly. In order to win, the answers of the players must also satisfy:

$$
\operatorname{rel}\left(g_{A}, g_{B}\right)=\operatorname{rel}\left(h_{A}, h_{B}\right)
$$

In other words, if Alice and Bob are given the same vertex, then they must respond with the same vertex. If they receive (non-)adjacent vertices they must return (non-)adjacent vertices. Also, assuming that Alice receives $g_{A}$ and Bob $h_{B}$, if Alice outputs $h_{A}=h_{B}$ then in order to win we require that Bob returns $g_{B}=g_{A}$. Note that we do not explicitly require that $G$ and $H$ have the same number of vertices. 


\subsection{Contributions}

In this work we use the $(G, H)$-isomorphism game in order to capture and extend the notion of graph isomorphism. First, we show that there exists a perfect classical strategy for the $(G, H)$ isomorphism game if and only if $G$ and $H$ are indeed isomorphic graphs. This suggests that by considering perfect quantum and non-signalling strategies for the $(G, H)$-isomorphism game we may define the notions of quantum and non-signalling isomorphisms of graphs.

When it comes to quantum strategies, there are two ways for Alice and Bob to perform measurements on separated systems. The first one is to assume a tensor product structure of the entire Hilbert space, and the second one is to assume that their measurement operators commute. In the former case we use the term "quantum strategy", whereas in the latter case, we use the term "quantum commuting strategy". The detailed formalism of quantum strategies and quantum commuting strategies will be given in Section 2 .

Definition 1.1. Two graphs $G$ and $H$ are quantum isomorphic/quantum commuting isomorphic/non-signalling isomorphic, denoted $G \cong_{q} H / G \cong_{q c} H / G \cong_{n s} H$, whenever there exists a perfect quantum/quantum commuting/non-signalling strategy for the $(G, H)$-isomorphism game.

This idea of associating a nonlocal game to a constraint satisfaction problem corresponding to a certain graph property and studying its quantum and non-signalling value is not new. This was first done for graph coloring in [9] and generalized to graph homomorphisms in [16].

Since every classical strategy can be considered as a quantum strategy, any quantum strategy can be considered as a quantum commuting strategy, and any quantum commuting strategy is non-signalling (see Equation (8)), we have that

$$
G \cong H \Longrightarrow G \cong_{q} H \Longrightarrow G \cong_{q c} H \Longrightarrow G \cong_{n s} H .
$$

As we will see, none of these implications can be reversed.

In Section 4 we focus on non-signalling isomorphism. Based on a result of Ramana, Scheinerman, and Ullman [28] which relates fractional isomorphism to the existence of a common equitable partition, in Theorem 4.5 we show the following:

Result 1. For any graphs $G$ and $H$ we have that $G \cong_{f} H$ if and only if $G \cong_{n s} H$.

The existence of a perfect non-signalling strategy for a general nonlocal game can be formulated as a (polynomially sized) linear program, and can thus be tested in polynomial time. However, by results of Tinhofer [33] and Babai, Erdős, and Selkow [6], fractional isomorphism can be decided in expected quadratic time (in the number of vertices). Thus Result 1 shows that for the class of isomorphism games, the existence of non-signalling strategies can be tested in expected quadratic time.

Combined with Result 1 this implies that non-signalling isomorphism is also polynomial-time decidable. Furthermore, it is known that fractional isomorphism (and thus also non-signalling isomorphism) distinguishes almost all graphs [6], and so it follows that the same holds for quantum commuting and quantum isomorphism, since they are more restrictive relations.

In Section 5 we focus on quantum isomorphism. We show that perfect quantum strategies for the isomorphism game must take a special form. This allows us to reformulate quantum isomorphism in terms of the existence of a set of projectors satisfying certain orthogonality constraints. Based on this we can show the following: 
Result 2. For any two graphs $G, H$ the following are equivalent:

(i) $G \cong{ }_{q} H$;

(ii) The system $\left(\overline{\mathrm{IQP}_{d}}\right)$ is feasible for some $d \in \mathbb{N}$;

(iii) The system $\left(\mathrm{ILP}_{d}\right)$ is feasible for some $d \in \mathbb{N}$.

Specifically, we prove the equivalence $(i) \Leftrightarrow(i i)$ in Theorem 5.4 and $(i) \Leftrightarrow(i i i)$ in Theorem 5.9 . As a consequence of Result 2 (iii) it follows that quantum isomorphic graphs must be cospectral with cospectral complements. This allows us to conclude that quantum and non-signalling isomorphism are different relations, since there are many examples of graphs that are fractionally isomorphic but not cospectral, e.g., any pair of $r$-regular graphs $G$ and $H$ are fractionally isomorphic since $A_{G} J=r J=J A_{H}$ where $J$ is the all ones matrix (which can be scaled to be doubly stochastic).

In Section 6 we consider the question of whether isomorphism and quantum isomorphism are different relations. In Theorem 6.4 we show that they are indeed distinct notions:

Result 3. There exist graphs that are quantum isomorphic but not isomorphic.

The main ingredient in the proof of Result 3 is a reduction from linear binary constraint system (BCS) games, introduced by Cleve and Mittal [11, to isomorphism games. Specifically, we show that a linear BCS game has a perfect classical (quantum) strategy if and only if a pair of graphs constructed from the BCS are (quantum) isomorphic. Since there exist linear BCS games that have perfect quantum strategies but no perfect classical strategies, this allows us to produce pairs of graphs that are quantum isomorphic but not isomorphic. The smallest example of such a pair we are able to construct uses the Mermin magic square game, which produces two graphs on 24 vertices each that are quantum isomorphic but not isomorphic (see Figures 1 and 2 ).

Lastly, in Section 7 we study whether quantum isomorphism and quantum commuting isomorphism are different relations. Using the same reduction as in Result 3, we obtain that a given linear BCS game has a perfect quantum commuting strategy if and only if the corresponding pair of graphs are quantum commuting isomorphic. Based on this reduction and a recent result of Slofstra [31, in Theorem 7.6 we show the following:

Result 4. There exist graphs that are quantum commuting isomorphic but not quantum isomorphic.

As a consequence of Result 4, quantum commuting isomorphism and non-signalling isomorphism are distinct relations, since the latter is decidable in polynomial-time by Result 1 .

Finally, since it has recently been shown that the existence of quantum/quantum commuting strategies for linear BCS games is undecidable [31, 32], our reduction implies the following:

Result 5. Determining if two graphs are quantum/quantum commuting isomorphic is undecidable.

\section{Preliminaries}

Linear algebra. The standard basis of $\mathbb{C}^{d}$ is denoted by $\left\{e_{i}: i \in[d]\right\}$, where $[d]:=\{1, \ldots, d\}$. For a matrix $X \in \mathbb{C}^{d \times d}$ we denote by $X^{\dagger}$ its conjugate transpose and by $X^{\top}$ its transpose. We denote the set of $d \times d$ Hermitian operators by $\mathcal{H}^{d}$. Throughout this work we equip $\mathcal{H}^{d}$ with the 
Hilbert-Schmidt inner product $\langle X, Y\rangle=\operatorname{Tr}\left(X^{\dagger} Y\right)$. A matrix $X \in \mathcal{H}^{d}$ is called positive semidefinite (psd) if $\psi^{\dagger} X \psi \geq 0$ for all $\psi \in \mathbb{C}^{d}$. The set of $d \times d$ psd matrices is denoted by $\mathcal{H}_{+}^{d}$. We use the fact that for two psd matrices $X, Y \in \mathcal{H}_{+}^{d}$ we have that $X Y=0$ if and only if $\langle X, Y\rangle=0$.

A matrix $E$ is called an (orthogonal) projector if it satisfies $E=E^{\dagger}=E^{2}$. We typically omit the term "orthogonal" because we will often refer to two projectors $E$ and $F$ being orthogonal (to each other) whenever they satisfy $E F=0$. We use the fact that for any family of projectors $\left\{E_{i}\right\}_{i}$ satisfying $\sum_{i} E_{i}=I$ we have that $E_{i} E_{j}=0$, for all $i \neq j$.

We denote by $\mathbb{M}_{n}(d)$ the set of $n \times n$ block matrices whose blocks are matrices in $\mathcal{H}^{d}$. For any family of matrices $\left\{E_{i j}\right\}_{i, j=1}^{n} \subseteq \mathcal{H}^{d}$ we denote by $\left[\left[E_{i j}\right]\right]$ the element of $\mathbb{M}_{n}(d)$ whose $(i, j)$-block is equal to $E_{i j}$. The $(i, j)$-block of a matrix $\mathcal{P} \in \mathbb{M}_{n}(d)$ is denoted by $\mathcal{P}_{i, j}$.

Quantum mechanics. In this section we briefly review some basic concepts from the theory of quantum information. For additional details we refer the reader to [22] and references therein.

To any quantum system one can associate a Hilbert space $\mathbb{C}^{d}$ (infinite dimensional spaces will be considered later). The state of the system is described by a unit vector $\psi \in \mathbb{C}^{d}$. Note that states that can be described in this way are actually known as pure states, and more generally the state of a quantum system is described by a Hermitian positive semidefinite matrix with trace equal to one. However, for quantum strategies for nonlocal games it suffices to consider only pure states, so we restrict our attention to this case.

One can obtain classical information from a quantum system by measuring it. For the purposes of this paper, the most relevant mathematical formalism of the concept of measurement is given by a Positive Operator-Valued Measure (POVM). A POVM $\mathcal{M}$ consists of a family of Hermitian psd matrices $\left\{M_{i} \in \mathcal{H}_{+}^{d}: i \in[m]\right\}$ such that $\sum_{i=1}^{m} M_{i}=I$, where $m$ is some integer and $I$ is the identity matrix. According to quantum mechanics, if the measurement $\mathcal{M}$ is performed on a quantum system in state $\psi \in \mathbb{C}^{d}$, then the probability that outcome $i$ occurs is $\psi^{\dagger} M_{i} \psi$. We say that a measurement $\mathcal{M}$ is projective if all of the POVM elements are projectors. It follows from the discussion in the "Linear algebra" paragraph that the POVM elements of any projective measurement are orthogonal to each other.

Consider two quantum systems $\mathrm{S}_{1}$ and $\mathrm{S}_{2}$ with corresponding state spaces $\mathbb{C}^{d_{1}}$ and $\mathbb{C}^{d_{2}}$ respectively. The state space of the joint system $\left(\mathrm{S}_{1}, \mathrm{~S}_{2}\right)$ is given by the tensor product $\mathbb{C}^{d_{1}} \otimes \mathbb{C}^{d_{2}}$. Moreover, if the system $\mathrm{S}_{1}$ is in (pure) state $\psi_{1} \in \mathbb{C}^{d_{1}}$ and $\mathrm{S}_{2}$ is in (pure) state $\psi_{2} \in \mathbb{C}^{d_{2}}$ then the joint system is in state $\psi_{1} \otimes \psi_{2} \in \mathbb{C}^{d_{1}} \otimes \mathbb{C}^{d_{2}}$. Not every state in the joint system space $\mathbb{C}^{d_{1}} \otimes \mathbb{C}^{d_{2}}$ can be written as a tensor product. States that cannot be written as a tensor product are known as entangled states.

If $\left\{M_{i} \in \mathcal{H}_{+}^{d_{1}}: i \in\left[m_{1}\right]\right\}$ and $\left\{N_{j} \in \mathcal{H}_{+}^{d_{2}}: j \in\left[m_{2}\right]\right\}$ define measurements on the individual systems $\mathrm{S}_{1}$ and $\mathrm{S}_{2}$ then the family of operators $\left\{M_{i} \otimes N_{j} \in \mathcal{H}_{+}^{d_{1} d_{2}}: i \in\left[m_{1}\right], j \in\left[m_{2}\right]\right\}$ defines a product measurement on the joint system $\left(\mathrm{S}_{1}, \mathrm{~S}_{2}\right)$. The probability of getting outcome $(i, j) \in$ $\left[m_{1}\right] \times\left[m_{2}\right]$, when measuring the quantum state $\psi$, is equal to $\psi^{*}\left(M_{i} \otimes N_{j}\right) \psi$.

It is often convenient to use the fact that any quantum state $\psi \in \mathbb{C}^{d} \otimes \mathbb{C}^{d}$ admits a so-called Schmidt decomposition: $\psi=\sum_{i=1}^{d} \lambda_{i} \alpha_{i} \otimes \beta_{i}$ where $\left\{\alpha_{i}: i \in[d]\right\}$ and $\left\{\beta_{i}: i \in[d]\right\}$ are orthonormal bases of $\mathbb{C}^{d}$ and $\lambda_{i} \geq 0$ for all $i \in[d]$. The bases $\left\{\alpha_{i}: i \in[d]\right\}$ and $\left\{\beta_{i}: i \in[d]\right\}$ are known as the Schmidt bases of $\psi$, and the $\lambda_{i}$ are its Schmidt coefficients. We say that $\psi$ has full Schmidt rank if its Schmidt coefficients are all positive. Note that one can also consider a Schmidt decomposition of states in $\mathbb{C}^{d_{1}} \otimes \mathbb{C}^{d_{2}}$ where $d_{1} \neq d_{2}$, but for us it suffices to consider $d_{1}=d_{2}$.

We say that a state is maximally entangled if it has full Schmidt rank and all of its Schmidt 
coefficients are the same. The canonical maximally entangled state in $\mathbb{C}^{d} \otimes \mathbb{C}^{d}$ is the state $\psi_{d}:=$ $\frac{1}{\sqrt{d}} \sum_{i=1}^{d} e_{i} \otimes e_{i}$, where $e_{i}$ is the $i^{\text {th }}$ standard basis vector. We will make use of the fact that

$$
\psi_{d}^{\dagger}(A \otimes B) \psi_{d}=\frac{1}{d} \operatorname{Tr}\left(A B^{\top}\right), \text { for all } A, B \in \mathbb{C}^{d \times d} .
$$

In the tensor product framework of quantum mechanics introduced above, each party has their own (finite dimensional) Hilbert space that they act on with positive operators. In the quantum commuting framework, both players share some, potentially infinite dimensional, Hilbert space $\mathcal{X}$ on which they both act with positive elements of the space of bounded linear operators on $\mathcal{X}$, denoted $\mathcal{B}(\mathcal{X})$. However, it is required that all of Alice's measurement operators commute with all of Bob's measurement operators. Thus if Alice performs the measurement $\left\{M_{i} \in \mathcal{B}(\mathcal{X})_{+}: i \in\left[m_{1}\right]\right\}$ and Bob performs the measurement $\left\{N_{j} \in \mathcal{B}(\mathcal{X})_{+}: j \in\left[m_{2}\right]\right\}$ on their shared state $\psi \in \mathcal{X}$, then it is required that $M_{i} N_{j}=N_{j} M_{i}$ for all $i \in\left[m_{1}\right], j \in\left[m_{2}\right]$, and the probability that they obtain outcome $(i, j)$ is given by $\psi^{\dagger} E_{i} F_{j} \psi$.

The quantum commuting framework is more general than the tensor product framework given above. To see this note that if $\left\{M_{i} \in \mathcal{H}_{+}^{d_{1}}: i \in\left[m_{1}\right]\right\}$ and $\left\{N_{j} \in \mathcal{H}_{+}^{d_{2}}: j \in\left[m_{2}\right]\right\}$ are measurements used by Alice and Bob in the tensor product framework, then the measurements $\left\{M_{i} \otimes I_{d_{2}} \in\right.$

$\left.\mathcal{H}_{+}^{d_{1}} \otimes \mathcal{H}_{+}^{d_{2}}: i \in\left[m_{1}\right]\right\}$ and $\left\{I_{d_{1}} \otimes N_{j} \in \mathcal{H}_{+}^{d_{1}} \otimes \mathcal{H}_{+}^{d_{2}}: j \in\left[m_{2}\right]\right\}$ are valid joint measurements in the quantum commuting framework that result in the same outcome probabilities.

It is also known, though it is nontrivial, that when restricted to finite dimensional Hilbert spaces, the two frameworks are equivalent [34, 30]. Thus we always allow for infinite dimensional Hilbert spaces when considering the quantum commuting framework.

\section{Strategies for the $(G, H)$-isomorphism game}

In this section we introduce three families of strategies for the $(G, H)$-isomorphism game (classical, quantum, and non-signalling) and study how they relate to each other.

Given a fixed strategy $S$ for the $(G, H)$-isomorphism game, we denote by $p_{S}\left(y_{A}, y_{B} \mid x_{A}, x_{B}\right)$ the joint conditional probability of Alice and Bob responding with $y_{A}$ and $y_{B}$ upon receiving inputs $x_{A}$ and $x_{B}$ respectively. We call such a joint conditional probability distribution a correlation. An easy but important observation is that $S$ is a perfect strategy if and only if $p_{S}\left(y_{A}, y_{B} \mid x_{A}, x_{B}\right)=0$ whenever $x_{A}, x_{B}, y_{A}, y_{B}$ do not meet the winning conditions of the game, i.e.,

$$
p_{S}\left(y_{A}, y_{B} \mid x_{A}, x_{B}\right)=0 \text {, when } \pi\left(x_{A}, x_{B}\right)>0 \text { and } V\left(x_{A}, x_{B}, y_{A}, y_{B}\right)=0 \text {. }
$$

In particular, if we specialize (4) to the $(G, H)$-isomorphism game we have that the correlation $p$ corresponds to a perfect strategy if and only if

$$
p\left(y_{A}, y_{B} \mid x_{A}, x_{B}\right)=0 \text {, whenever conditions (1) or (2) fail. }
$$

As a consequence we have that any winning strategy for the $(G, H)$-isomorphism game is also a winning strategy for the $(H, G)$-isomorphism game, as well as the $(\bar{G}, \bar{H})$-isomorphism game, where $\bar{G}$ and $\bar{H}$ denote the complements of $G$ and $H$ respectively. 


\subsection{Classical Strategies}

In a classical strategy, Alice and Bob are allowed to make use of shared randomness to determine how they respond. Note that this does not allow them to communicate. They may also use local randomness, but this can be incorporated into the shared randomness without loss of generality.

Lemma 3.1. The $(G, H)$-isomorphism game admits a perfect classical strategy if and only if $G$ and $H$ are isomorphic.

Proof. Suppose that $\varphi: V(G) \rightarrow V(H)$ is an isomorphism of $G$ and $H$. It is easy to see that if both players respond with $\varphi(g)$ upon receiving $g \in V(G)$ and with $\varphi^{-1}(h)$ upon receiving $h \in V(H)$, they win the $(G, H)$-isomorphism game with certainty.

Conversely, assume that the $(G, H)$-isomorphism game admits a perfect classical strategy $S$. First, we show that there also exists a perfect deterministic classical strategy. The correlation corresponding to $S$, denoted by $p$, can be decomposed as a convex combination $p=\sum_{i} \lambda_{i} p_{i}$, where the $\lambda_{i}$ 's encode the shared randomness and the $p_{i}$ 's are correlations arising from deterministic classical strategies. Since they are deterministic, each of the $p_{i}$ satisfy $p_{i}\left(y_{A}, y_{B} \mid x_{A}, x_{B}\right) \in\{0,1\}$ for all $x_{A}, x_{B}, y_{A}, y_{B} \in V(G) \cup V(H)$. Since the correlation $p$ corresponds to a perfect strategy, it must satisfy (5). But this happens if and only if (5) is satisfied by all of the deterministic classical correlations $p_{i}$ that appear in the convex combination $p=\sum_{i} \lambda_{i} p_{i}$.

A deterministic classical strategy is a pair of functions, $f_{A}, f_{B}: V(G) \cup V(H) \rightarrow V(G) \cup V(H)$, which map inputs to outputs for each of Alice and Bob respectively. Assuming the strategy is winning, we have that $f_{A}(x), f_{B}(x) \in V(G) \Leftrightarrow x \in V(H)$, and that $f_{A}(x)=f_{B}(x)$ for all $x \in$ $V(G) \cup V(H)$. Since $f_{A}=f_{B}$, we will refer to both of them as simply $f$. For $g, g^{\prime} \in V(G)$, the winning conditions of the $(G, H)$-isomorphism game require that $\operatorname{rel}\left(g, g^{\prime}\right)=\operatorname{rel}\left(f(g), f\left(g^{\prime}\right)\right)$. This implies that the restriction of $f$ to $V(G)$ is an isomorphism from $G$ to an induced subgraph of $H$. Similarly, the restriction of $f$ to $V(H)$ is an isomorphism of $H$ to an induced subgraph of $G$. This is only possible if $G$ and $H$ are isomorphic and the above two restrictions of $f$ are isomorphisms. Finally, for $g^{*} \in V(G)$, let $h^{*}=f\left(g^{*}\right)$. The case where Alice is sent $g^{*}$ and Bob is sent $h^{*}$ allows us to conclude that $\operatorname{rel}\left(g^{*}, f\left(h^{*}\right)\right)=\operatorname{rel}\left(f\left(g^{*}\right), h^{*}\right)$. Since $h^{*}=f\left(g^{*}\right)$, the relationship between these vertices is "equality", thus $g^{*}=f\left(h^{*}\right)$. In other words, the restriction $\left.f\right|_{V(G)}$ is the inverse of the restriction $\left.f\right|_{V(H)}$. Thus, any perfect deterministic strategy for the $(G, H)$-isomorphism game corresponds to Alice and Bob responding according to a fixed isomorphism between $G$ and $H$.

\subsection{Quantum Strategies}

A quantum strategy for the $(G, H)$-isomorphism game consists of a shared entangled state $\psi$, and POVMs $\mathcal{E}_{x}=\left\{E_{x y}: y \in V(G) \cup V(H)\right\}$ for each $x \in V(G) \cup V(H)$ for Alice, and $\mathcal{F}_{x}=\left\{F_{x y}\right.$ : $y \in V(G) \cup V(H)\}$ for each $x \in V(G) \cup V(H)$ for Bob. Upon receiving $x_{A} \in V(G) \cup V(H)$ Alice performs measurement $\mathcal{E}_{x_{A}}$ and obtains some outcome $y_{A} \in V(G) \cup V(H)$. Similarly, upon receiving $x_{B}$ Bob measures $\mathcal{F}_{x_{B}}$ and obtains some $y_{B}$. The probability of Alice and Bob outputting vertices $y_{A}$ and $y_{B}$ upon receiving $x_{A}$ and $x_{B}$ respectively is given by

$$
p\left(y_{A}, y_{B} \mid x_{A}, x_{B}\right)=\psi^{\dagger}\left(E_{x_{A} y_{A}} \otimes F_{x_{B} y_{B}}\right) \psi .
$$

Any correlation that can be realized as in (6) is known as a quantum correlation.

We remark that Alice and Bob only need to make one measurement each, and that it does not matter who measures first. The former claim follows because any series of measurements made by 
one of the parties can be combined into a single measurement, and the latter follows because Alice and Bob's measurements commute.

Therefore, it follows by (5) that a quantum strategy as described above is a winning strategy for the $(G, H)$-isomorphism game if and only if

$$
\psi^{\dagger}\left(E_{x_{A} y_{A}} \otimes F_{x_{B} y_{B}}\right) \psi=0 \text {, whenever conditions (1) or (2) fail. }
$$

A quantum isomorphism is a quantum strategy for the $(G, H)$-isomorphism game satisfying (7).

It is important to note that any classical correlation is also a quantum correlation. Indeed, any deterministic strategy can be produced by using measurements in which all but one of the POVM elements is the zero matrix. The remaining POVM element will be the identity and performing this measurement will always result in the outcome corresponding to the identity. Since any classical shared randomness can also be replicated by measurements on a shared state, this shows that any classical correlation can be produced by some quantum strategy.

A quantum commuting strategy for the $(G, H)$-isomorphism game consists of a (potentially infinite-dimensional) Hilbert space $\mathcal{X}$, a unit vector $\psi \in \mathcal{X}$, and measurement operators that correspond to bounded positive linear operators acting on $\mathcal{X}$, such that Alice's measurement operators commute with all of Bob's measurement operators. A quantum commuting strategy is a winning strategy for the $(G, H)$-isomorphism game if and only if (7) holds but with the tensor product replaced with the usual product of operators.

It was recently shown by Slofstra [31] that the quantum commuting framework allows to win some nonlocal games that cannot be perfectly won using the tensor product framework. In Section 6 we will use this result to show that this also holds for the graph isomorphism game.

\subsection{Non-signalling Strategies}

Suppose that Alice and Bob are playing a nonlocal game with a quantum strategy as described in the previous section. If Alice is given input $x_{A}$, and Bob is given input $x_{B}$, the probability that Alice obtains outcome $y_{A}$ when she performs measurement $\mathcal{E}_{x_{A}}$ is given by:

$$
\sum_{y_{B}} p\left(y_{A}, y_{B} \mid x_{A}, x_{B}\right)=\sum_{y_{B}} \psi^{\dagger}\left(E_{x_{A} y_{A}} \otimes F_{x_{B} y_{B}}\right) \psi=\psi^{\dagger}\left(E_{x_{A} y_{A}} \otimes I\right) \psi
$$

and we see that this does not depend on Bob's input $x_{B}$. Similarly, the probability of Bob obtaining a particular outcome will not be dependent on Alice's input. This property of quantum correlations is known as the non-signalling property. Formally, a correlation $p\left(y_{A}, y_{B} \mid x_{A}, x_{B}\right)$ is non-signalling if

$$
\begin{aligned}
& \sum_{y_{B}} p\left(y_{A}, y_{B} \mid x_{A}, x_{B}\right)=\sum_{y_{B}} p\left(y_{A}, y_{B} \mid x_{A}, x_{B}^{\prime}\right), \text { for all } x_{A}, y_{A}, x_{B}, x_{B}^{\prime}, \text { and } \\
& \sum_{y_{A}} p\left(y_{A}, y_{B} \mid x_{A}, x_{B}\right)=\sum_{y_{A}} p\left(y_{A}, y_{B} \mid x_{A}^{\prime}, x_{B}\right), \text { for all } x_{B}, y_{B}, x_{A}, x_{A}^{\prime} .
\end{aligned}
$$

A correlation which is signalling allows Alice and Bob to communicate some information. However, this violates the definition of a nonlocal game, since one of the requirements is that the players are not allowed to communicate during the game. Thus, one of the reasons for considering nonsignalling correlations is that they represent the largest class of admissible correlations for nonlocal 
games. More practically, since the non-signalling condition is linear, these correlations often provide tractable upper bounds on the power of quantum correlations. Indeed, in the next section we will see that we can completely characterize when two graphs are non-signalling isomorphic.

Furthermore, as we have seen, all quantum correlations are non-signalling. However, the converse is not true. For instance, for input and output sets equal to $\{0,1\}$, the correlation given by:

$$
p\left(y, y^{\prime} \mid x, x^{\prime}\right)= \begin{cases}\frac{1}{2}, & \text { if } y+y^{\prime} \equiv x x^{\prime} \bmod 2 \\ 0, & \text { otherwise }\end{cases}
$$

is known as the $P R$ box [27]. One can check that the $\mathrm{PR}$ box is non-signalling, but it is well known [27] that it cannot be implemented by any quantum strategy.

A general non-signalling correlation may not be physically realizable, so when we refer to nonsignalling strategies, we can think of this as Alice and Bob each simply having some black box where they enter their inputs into and which gives them their outputs. We only require that the resulting correlation produced by these boxes obeys the non-signalling condition.

\section{Non-signalling Isomorphism}

Our goal in this section is to show Result 1, i.e., that fractional isomorphism and non-signalling isomorphism are equivalent relations.

\subsection{Non-signalling isomorphism implies fractional isomorphism}

To show that non-signalling isomorphism implies fractional isomorphism we show that one can use a non-signalling correlation that wins the $(G, H)$-isomorphism game to construct a doubly stochastic matrix $D$ satisfying $A_{G} D=D A_{H}$. To do this we will need to perform several manipulations of correlation probabilities. In an effort to make things more readable, we will make use of some conventions. We will typically use $g, g^{\prime}$, etc., for vertices of a graph $G$, and similarly use $h, h^{\prime}$, etc., for vertices of $H$. For elements of $V:=V(G) \cup V(H)$ whose membership in $V(G)$ or $V(H)$ is ambiguous, we will use $x$ or $y$ or some variant thereof. Also, we will try to use $x$ as an input and $y$ as an output whenever possible.

First, if $p$ is a winning non-signalling correlation for the $(G, H)$-isomorphism game, then we must have that $p\left(g, y \mid g^{\prime}, x\right)=0$ whenever $g, g^{\prime} \in V(G)$, and similarly when we replace $G$ by $H$ and/or switch Alice and Bob's positions. Furthermore, for all $h \in V(H)$ we have that $p\left(g, g^{\prime} \mid h, h\right)=0$ if $g \neq g^{\prime}$, and similarly with $H$ replaced by $G$. Therefore, we have the following observation:

$$
\sum_{h^{\prime} \in V(H)} p\left(h^{\prime}, h^{\prime} \mid g, g\right)=\sum_{g^{\prime} \in V(G)} p\left(g^{\prime}, g^{\prime} \mid h, h\right)=1, \text { for all } g \in V(G), h \in V(H) .
$$

Our goal is to use 10 to construct the desired doubly stochastic matrix. Specifically, the above sums will correspond to its row and column sums. We need the following intermediate result.

Lemma 4.1. Let $p$ be a winning non-signalling correlation for the $(G, H)$-isomorphism game. Then,

$$
p(h, h \mid g, g)=p(g, h \mid h, g)=p(h, g \mid g, h)=p(g, g \mid h, h),
$$

for all $g \in V(G), h \in V(H)$. 
Proof. Set $V:=V(G) \cup V(H)$. For all $g \in V(G)$ and $h \in V(H)$ we have that

$$
p(h, h \mid g, g)=\sum_{y \in V} p(h, y \mid g, g)=\sum_{y \in V} p(h, y \mid g, h)=p(h, g \mid g, h),
$$

where we use (5) for the first equality, for the second equality we use that $p$ is non-signalling and for the third equality we again use (5). Similarly, we get that

$$
p(h, h \mid g, g)=\sum_{y \in V} p(y, h \mid g, g)=\sum_{y \in V} p(y, h \mid h, g)=p(g, h \mid h, g) \text {. }
$$

Lastly, by the symmetry of $G$ and $H$ we also have that $p(g, g \mid h, h)=p(g, h \mid h, g)$. Putting everything together the lemma follows.

Note that by combining Lemma 4.1 with Equation 100 we get that

$$
|V(G)|=\sum_{g \in V(G), h \in V(H)} p(h, h \mid g, g)=\sum_{g \in V(G), h \in V(H)} p(g, g \mid h, h)=|V(H)|,
$$

which was not obvious even for quantum strategies.

We can now show that two graphs which are non-signalling isomorphic are necessarily fractionally isomorphic.

Lemma 4.2. If $G$ and $H$ are non-signalling isomorphic, then they are fractionally isomorphic.

Proof. Set $V:=V(G) \cup V(H)$ and let $A_{G}$ and $A_{H}$ be the adjacency matrices of $G$ and $H$ respectively. Define $D$ to be a matrix with rows indexed by $V(G)$ and columns by $V(H)$ such that $D_{g h}=$ $p(h, h \mid g, g)$, for all $g \in V(G), h \in V(H)$. We show that $D$ is doubly stochastic and satisfies $A_{G} D=D A_{H}$. First, the $g^{\text {th }}$ row sum of $D$ is given by $\sum_{h \in V(H)} p(h, h \mid g, g)$ which is equal to 1 by Equation 10 . Furthermore, the $h^{\text {th }}$ column sum of $D$ is given by:

$$
\sum_{g \in V(G)} p(h, h \mid g, g)=\sum_{g \in V(G)} p(g, g \mid h, h)=1
$$

where for the first equality we use Lemma 4.1 and for the second one we use (10). Since the entries of $D$ are also obviously nonnegative, we have that $D$ is doubly stochastic.

Consider the $(g, h)$-entries of the matrices $A_{G} D$ and $D A_{H}$. We have that

$$
\begin{aligned}
& \left(A_{G} D\right)_{g h}=\sum_{g^{\prime}: g^{\prime} \sim g} p\left(h, h \mid g^{\prime}, g^{\prime}\right) \\
& \left(D A_{H}\right)_{g h}=\sum_{h^{\prime}: h^{\prime} \sim h} p\left(h^{\prime}, h^{\prime} \mid g, g\right),
\end{aligned}
$$

where $\sim$ denotes adjacency. Making repeated use of (5) and the non-signalling conditions (9) we 
get that

$$
\begin{aligned}
\sum_{h^{\prime}: h^{\prime} \sim h} p\left(h^{\prime}, h^{\prime} \mid g, g\right) & =\sum_{h^{\prime}: h^{\prime} \sim h} \sum_{y \in V} p\left(h^{\prime}, y \mid g, g\right)=\sum_{h^{\prime}: h^{\prime} \sim h} \sum_{y \in V} p\left(h^{\prime}, y \mid g, h\right) \\
& =\sum_{h^{\prime}: h^{\prime} \sim h} \sum_{g^{\prime}: g^{\prime} \sim g} p\left(h^{\prime}, g^{\prime} \mid g, h\right)=\sum_{g^{\prime}: g^{\prime} \sim g} \sum_{h^{\prime}: h^{\prime} \sim h} p\left(h^{\prime}, g^{\prime} \mid g, h\right) \\
& =\sum_{g^{\prime}: g^{\prime} \sim g} \sum_{y \in V} p\left(y, g^{\prime} \mid g, h\right)=\sum_{g^{\prime}: g^{\prime} \sim g} \sum_{y \in V} p\left(y, g^{\prime} \mid h, h\right) \\
& =\sum_{g^{\prime}: g^{\prime} \sim g} p\left(g^{\prime}, g^{\prime} \mid h, h\right) .
\end{aligned}
$$

By Lemma 4.1 we have $p\left(g^{\prime}, g^{\prime} \mid h, h\right)=p\left(h, h \mid g^{\prime}, g^{\prime}\right)$ and thus (11) implies that

$$
\sum_{h^{\prime}: h^{\prime} \sim h} p\left(h^{\prime}, h^{\prime} \mid g, g\right)=\sum_{g^{\prime}: g^{\prime} \sim g} p\left(h, h \mid g^{\prime}, g^{\prime}\right),
$$

i.e., that $\left(D A_{H}\right)_{g h}=\left(A_{G} D\right)_{g h}$. Therefore $A_{G} D=D A_{H}$ and $G$ and $H$ are fractionally isomorphic.

\subsection{Fractional isomorphism implies non-signalling isomorphism}

To show the converse of Lemma 4.2, we use a result of Ramana, Scheinerman, and Ullman [28]. To explain this result we first need to introduce some definitions. Let $\mathcal{C}=\left\{C_{1}, \ldots, C_{k}\right\}$ be a partition of $V(G)$ for some graph $G$. The partition $\mathcal{C}$ is called equitable if there exist numbers $c_{i j}$ for $i, j \in[k]$ such that any vertex in $C_{i}$ has exactly $c_{i j}$ neighbors in $C_{j}$. Note that $c_{i j}$ and $c_{j i}$ are not necessarily equal, but $c_{i j}\left|C_{i}\right|=c_{j i}\left|C_{j}\right|$. We refer to the numbers $c_{i j}$ as the partition numbers and to the sets $C_{i}$ as the cells of an equitable partition $\mathcal{C}=\left\{C_{1}, \ldots, C_{k}\right\}$. A trivial example of this is the partition where each part has size 1. Less trivially, if $G$ is regular, the partition with only one cell is equitable.

Equivalently, a partition $\mathcal{C}=\left\{C_{1}, \ldots, C_{k}\right\}$ is equitable if for any $i \in[k]$, the subgraph induced by the vertices in $C_{i}$ is regular, and for any $i \neq j \in[k]$ the subgraph with vertex set $C_{i} \cup C_{j}$ and containing the edges between $C_{i}$ and $C_{j}$ is a semiregular bipartite graph, i.e., a bipartite graph for which every two vertices on the same bipartition have the same degree.

We say that $\mathcal{C}$ and $\mathcal{D}$ have a common equitable partition if there exist equitable partitions $\mathcal{C}=\left\{C_{1}, \ldots, C_{k}\right\}$ and $\mathcal{D}=\left\{D_{1}, \ldots, D_{k^{\prime}}\right\}$ for $G$ and $H$ respectively, satisfying $k=k^{\prime},\left|C_{i}\right|=\left|D_{i}\right|$ for all $i \in[k]$, and lastly, $c_{i j}=d_{i j}$ for all $i, j \in[k]$. As it turns out, common equitable partitions characterize the notion of fractional isomorphism. Specifically:

Theorem 4.3 ([28]). Two graphs are fractionally isomorphic if and only if they have a common equitable partition.

As an example, if $G$ and $H$ are $d$-regular with the same number of vertices, then the single cell partitions form a common equitable partition, and thus any such graphs are fractionally isomorphic.

We prove the converse of Lemma 4.2 by showing that a common equitable partition can be used to construct a non-signalling correlation that wins the $(G, H)$-isomorphism game.

Lemma 4.4. If $G$ and $H$ are fractionally isomorphic, then they are non-signalling isomorphic. 
Proof. As $G \cong_{f} H$, by Theorem 4.3 the graphs $G$ and $H$ have a common equitable partition $\mathcal{C}=\left\{C_{1}, \ldots, C_{k}\right\}$ and $\mathcal{D}=\left\{D_{1}, \ldots, D_{k}\right\}$ respectively. Let $n_{i}=\left|C_{i}\right|=\left|D_{i}\right|$ for all $i$ and let $c_{i j}$ for $i, j \in[k]$ be the common partition numbers. Also define $\bar{c}_{i j}:=n_{j}-c_{i j}-\delta_{i j}$, where $\delta_{i j}$ is the Kronecker delta function. Note that $\bar{c}_{i j}$ is the number of non-neighbors a vertex of $C_{i}$ has in $C_{j}$.

We use this common equitable partition to construct a winning non-signalling correlation $p$. The idea is roughly that if Alice and Bob are given $g \in C_{i}$ and $g^{\prime} \in C_{j}$ respectively such that $g \sim g^{\prime}$, they should respond in a correlated manner with the endpoints of a randomly chosen edge between $D_{i}$ and $D_{j}$. Formally, for $g \in C_{i}, g^{\prime} \in C_{j}, h \in D_{i}, h^{\prime} \in D_{j}$, define a correlation $p$ as follows:

$$
p\left(h, h^{\prime} \mid g, g^{\prime}\right)= \begin{cases}\frac{1}{n_{i} c_{i j}}, & \text { if } g \sim g^{\prime} \& h \sim h^{\prime} \\ \frac{1}{n_{i} \overline{c_{i j}}}, & \text { if } g \neq g^{\prime} \& h \neq h^{\prime} \\ \frac{1}{n_{i}}, & \text { if } g=g^{\prime} \& h=h^{\prime} \\ 0, & \text { otherwise }\end{cases}
$$

where $\nsucceq$ denotes that the vertices are distinct and non-adjacent. Furthermore, define

$$
p\left(h, h^{\prime} \mid g, g^{\prime}\right)=p\left(h, g^{\prime} \mid g, h^{\prime}\right)=p\left(g, h^{\prime} \mid h, g^{\prime}\right)=p\left(g, g^{\prime} \mid h, h^{\prime}\right), \forall g, g^{\prime}, h, h^{\prime},
$$

and lastly set $p\left(y, y^{\prime} \mid x, x^{\prime}\right)$ equal to zero for all values of $x, x^{\prime}, y, y^{\prime}$ not yet accounted for.

It is easy to verify that this correlation evaluates to zero when Alice and Bob's inputs and outputs do not meet the winning conditions of the $(G, H)$-isomorphism game and thus it corresponds to a perfect strategy. Thus, it only remains to show that $p$ is a valid non-signalling correlation, i.e., it satisfies the equations in (9). In fact, we show that for all $x_{A}, x_{B}, y_{A} \in V(G) \cup V(H)$ we have:

$$
\sum_{y_{B} \in V} p\left(y_{A}, y_{B} \mid x_{A}, x_{B}\right)= \begin{cases}\frac{1}{n_{i}}, & \text { if } \exists i \in[k] \text { where }\left(x_{A} \in C_{i} \& y_{A} \in D_{i}\right) \text { or }\left(x_{A} \in D_{i} \& y_{A} \in C_{i}\right) ; \\ 0, & \text { otherwise. }\end{cases}
$$

and similarly with Alice and Bob exchanged. As this does not depend on the choice of $x_{B}$ it follows by definition that the correlation $p$ is non-signalling.

Now we proceed to prove (14). If $x_{A} \in C_{i} \& y_{A} \notin D_{i}$ (or $x_{A} \in D_{i} \& y_{A} \notin C_{i}$ ) we have by definition that $\sum_{y_{B}} p\left(y_{A}, y_{B} \mid x_{A}, x_{B}\right)=0$. It remains to consider the case $g:=x_{A} \in C_{i}$ and $h:=y_{A} \in D_{i}$ (the case $x_{A} \in D_{i} \& y_{A} \in C_{i}$ follows similarly). For clarity of exposition we divide the proof in two subcases.

Case 1: If $g^{\prime}:=x_{B} \in C_{j}$ it follows by 12 that

$$
\sum_{y_{B} \in V} p\left(h, y_{B} \mid g, g^{\prime}\right)=\sum_{h^{\prime} \in D_{j}} p\left(h, h^{\prime} \mid g, g^{\prime}\right)= \begin{cases}p\left(h, h \mid g, g^{\prime}\right), & \text { if } g=g^{\prime} ; \\ \sum_{h^{\prime} \in D_{j} \cap N(h)} p\left(h, h^{\prime} \mid g, g^{\prime}\right), & \text { if } g \sim g^{\prime} ; \\ \sum_{h^{\prime} \in D_{j} \cap N[h]^{c}} p\left(h, h^{\prime} \mid g, g^{\prime}\right), & \text { if } g \not g^{\prime},\end{cases}
$$

where $N(h)$ and $N[h]$, denote the open and closed neighbourhoods of $h$ in $H$ respectively, and $N[h]^{c}$ denotes the complement of $N[h]$ in $V(H)$. Again by Equation 12 this evaluates to $1 / n_{i}$ in all three cases. 
Case 2: If $h^{\prime}:=x_{B} \in D_{j}$ we have that:

$$
\sum_{y_{B} \in V} p\left(h, y_{B} \mid g, h^{\prime}\right)=\sum_{g^{\prime} \in C_{j}} p\left(h, g^{\prime} \mid g, h^{\prime}\right)=\sum_{g^{\prime} \in C_{j}} p\left(g, g^{\prime} \mid h, h^{\prime}\right)=1 / n_{i},
$$

where the first equality follows from $(12)$, the second one from $(13)$ and the third one by Case 1 .

Lastly, we show that $p$ is a valid probability distribution. For this, let $x_{A}, x_{B} \in V$ and assume that $x_{A} \in C_{i}$ (the case $x_{A} \in D_{i}$ is similar). Then, we have that

$$
\sum_{y_{A} \in V} \sum_{y_{B} \in V} p\left(y_{A}, y_{B} \mid x_{A}, x_{B}\right)=\sum_{y_{A} \in D_{i}} \sum_{y_{B} \in V} p\left(y_{A}, y_{B} \mid x_{A}, x_{B}\right)=\sum_{y_{A} \in D_{i}} \frac{1}{n_{i}}=1,
$$

where for the second equality we used (14).

Combining Lemma 4.2 with Lemma 4.4 we immediately get Result 1.

Theorem 4.5. For any graphs $G$ and $H$ we have that $G \cong_{f} H$ if and only if $G \cong_{n s} H$.

As mentioned above, a common example of graphs that are fractionally isomorphic but not isomorphic is any pair of non-isomorphic $d$-regular graphs on $n$ vertices. This makes it seem like fractional isomorphism is a quite coarse relaxation of isomorphism. But in fact it is known [6] that asymptotically almost surely every graph is not fractionally isomorphic to any graph that it is not also isomorphic to. More precisely, if one picks a labelled $n$-vertex graph uniformly at random, then the probability that this graph is fractionally isomorphic to a graph that it is non-isomorphic to goes to zero as $n$ goes to infinity. Standard results imply that the same holds if an unlabelled graph (i.e., an isomorphism class of graphs) is chosen at random. Since non-signalling/fractional isomorphism is the coarsest relation we will consider in this work, the same holds for all the other relations we will see.

It is worth noting that this is quite different from the related graph-based nonlocal game known as the $(G, H)$-homomorphism game [16, 29]. As its name suggests, this game can be won classically if and only if there exists a homomorphism (adjacency-preserving map) from $G$ to $H$. However, if non-signalling strategies are allowed, the game becomes trivial: it can always be won as long as $H$ has at least one edge [16].

\section{Quantum graph isomorphism}

In this section we prove Result 2, i.e., we show that quantum isomorphism coincides with existence of feasible solutions to the programs $\left(\overline{\mathrm{IQP}_{d}}\right)$ and $\left(\overline{\mathrm{ILP}_{d}}\right)$. First, we introduce a useful mathematical tool. Let vec : $\mathbb{C}^{d_{1} \times d_{2}} \rightarrow \mathbb{C}^{d_{1}} \otimes \mathbb{C}^{d_{2}}$ be the linear map that takes the matrix $u v^{\dagger}$ to $u \otimes \bar{v}$, where $\bar{v}$ denotes the entrywise complex conjugate of $v$. In other words, the map vec creates a vector from a matrix by stacking (the transpose of) its rows on top of each other. Also, let mat : $\mathbb{C}^{d_{1}} \otimes \mathbb{C}^{d_{2}} \rightarrow \mathbb{C}^{d_{1} \times d_{2}}$ be the inverse of the vectorization map. It is not hard to see that the vec map is an isometry. Setting $\rho=\operatorname{mat}(\psi)$ we have that

$$
\psi^{\dagger}(E \otimes F) \psi=\operatorname{vec}(\rho)^{\dagger}(E \otimes F) \operatorname{vec}(\rho)=\operatorname{vec}(\rho)^{\dagger} \operatorname{vec}\left(E \rho F^{\mathbf{T}}\right)=\operatorname{Tr}\left(\rho^{\dagger} E \rho F^{\mathbf{T}}\right),
$$

where we used the fact that the vec map is an isometry and the identity $\operatorname{vec}\left(A X B^{\top}\right)=(A \otimes$ $B) \operatorname{vec}(X)$, for Hermitian operators of appropriate size. This identity is crucial for our results in this section. 


\subsection{Common techniques for analyzing quantum strategies}

Recall that a correlation $p\left(y, y^{\prime} \mid x, x^{\prime}\right)$ is quantum if it can be generated by a quantum strategy, i.e., if there exists a quantum state $\psi \in \mathbb{C}^{d} \otimes \mathbb{C}^{d}$ and measurements $\left\{E_{x y}\right\}_{y}$ and $\left\{F_{x y}\right\}_{y}$ such that

$$
p\left(y, y^{\prime} \mid x, x^{\prime}\right)=\psi^{\dagger}\left(E_{x y} \otimes F_{x^{\prime} y^{\prime}}\right) \psi, \forall x, x^{\prime}, y, y^{\prime} .
$$

It is well-known that we may assume without loss of generality that the state $\psi$ has full Schmidt rank. To see this let $\psi=\sum_{i=1}^{d^{\prime}} \lambda_{i} \alpha_{i} \otimes \beta_{i}$ be the Schmidt decomposition for $\psi$, where $\left\{\alpha_{i}\right\}_{i=1}^{d^{\prime}}$ and $\left\{\beta_{i}\right\}_{i=1}^{d^{\prime}}$ are orthonormal families of vectors in $\mathbb{C}^{d}$ and $\lambda_{i}>0$ for all $i \in\left[d^{\prime}\right]$. Consider the isometries $U_{A}=\sum_{i=1}^{d^{\prime}} e_{i} \alpha_{i}^{\dagger}$ and $U_{B}=\sum_{i=1}^{d^{\prime}} e_{i} \beta_{i}^{\dagger}$, where $\left\{e_{i}\right\}_{i=1}^{d^{\prime}} \subseteq \mathbb{C}^{d^{\prime}}$ is the standard orthonormal basis of $\mathbb{C}^{d^{\prime}}$. Define

$$
\tilde{\psi}=\left(U_{A} \otimes U_{B}\right) \psi, \quad \tilde{E}_{x y}=U_{A} E_{x y} U_{A}^{\dagger}, \forall x, y \quad \text { and } \quad \tilde{F}_{x y}=U_{B} F_{x y} U_{B}^{\dagger}, \forall x, y .
$$

It is easy to verify that the matrices $\left\{\tilde{E}_{x y}\right\}_{y}$ and $\left\{\tilde{F}_{x y}\right\}_{y}$ form valid quantum measurements and

$$
\tilde{\psi}=\sum_{i=1}^{d^{\prime}} \lambda_{i} e_{i} \otimes e_{i} \in \mathbb{C}^{d^{\prime}} \otimes \mathbb{C}^{d^{\prime}},
$$

is a valid quantum state with full Schmidt rank. Furthermore, the quantum strategy corresponding to $\tilde{\psi},\left\{\tilde{E}_{x y}\right\}_{y}$ and $\left\{\tilde{F}_{x y}\right\}$ also generates the correlation $p\left(y, y^{\prime} \mid x, x^{\prime}\right)$ given in (18). Another useful consequence of this fact is that the operator $\rho=\operatorname{mat}(\tilde{\psi})$ is a diagonal matrix with positive entries. When considering quantum strategies for the isomorphism game, we will often say we are "working in the Schmidt basis of the shared state $\psi$ ". By this we mean that we have implicitly performed the above transformation on the shared state and measurement operators of our strategy.

\subsection{Characterizing perfect quantum strategies}

In Section 3.2 we described the general form of a quantum strategy for the $(G, H)$-isomorphism game. In this section we investigate quantum isomorphisms in more detail, and show that perfect quantum strategies can always be chosen to take a specific form.

Definition 5.1. A nonlocal game is called synchronous if the players share the same question set $X$, the same answer set $Y$, and furthermore,

$$
V\left(y, y^{\prime} \mid x, x\right)=0, \text { for all } x \in X \text { and } y \neq y^{\prime} \in Y \text {. }
$$

Analogously, a correlation is called synchronous if $p\left(y, y^{\prime} \mid x, x\right)=0$, for all $x \in X$ and $y \neq y^{\prime} \in Y$.

For example, note that the $(G, H)$-isomorphism game is synchronous. Indeed, in this game the question and answer sets are both equal to $V(G) \cup V(H)$. Furthermore, if the players are given the same vertex and they respond with different vertices they lose. This shows that (20) is satisfied.

Synchronous games have recently received significant attention in the literature due to the fact that their perfect quantum strategies always have a special form. Specifically, the following result or a similar version has appeared in various places [16, 29, 17, 9].

Lemma 5.2. Let $\psi \in \mathbb{C}^{d} \otimes \mathbb{C}^{d}, \mathcal{E}_{x}=\left\{E_{x y}: y \in Y\right\}$, and $\mathcal{F}_{x}=\left\{F_{x y}: y \in Y\right\}$ for all $x \in X$ be $a$ perfect quantum strategy for a synchronous game. If $\psi$ and the operators $E_{x y}$ and $F_{x y}$ are expressed in the Schmidt basis of $\psi$, and $\rho=\operatorname{mat}(\psi)$, then 
(i) $E_{x y}=F_{x y}^{\top}$ for all $x \in X, y \in Y$;

(ii) $E_{x y}$ and $F_{x y}$ are projectors for all $x \in X, y \in Y$;

(iii) $E_{x y} \rho=\rho E_{x y}$ and $F_{x y} \rho=\rho F_{x y}$ for all $x \in X, y \in Y$;

(iv) $p\left(y, y^{\prime} \mid x, x^{\prime}\right)=\psi^{\dagger}\left(E_{x y} \otimes F_{x^{\prime} y^{\prime}}\right) \psi=0$ if and only if $E_{x y} E_{x^{\prime} y^{\prime}}=0$.

Since the graph isomorphism game is synchronous, Lemma 5.2 applies to it. However, in our next result we show that even more conditions are met by perfect quantum strategies for the isomorphism game.

Theorem 5.3. Consider two graphs $G$ and $H$ and set $V=V(G) \cup V(H)$. Let $\psi \in \mathbb{C}^{d} \otimes \mathbb{C}^{d}$, $\mathcal{E}_{x}=\left\{E_{x y}: y \in V\right\}$, and $\mathcal{F}_{x}=\left\{F_{x y}: y \in V\right\}$ for all $x \in V$ be a perfect quantum strategy for the $(G, H)$-isomorphism game. If $\psi$ and the operators $E_{x y}$ and $F_{x y}$ are expressed in the Schmidt basis of $\psi$, and $\rho=\operatorname{mat}(\psi)$, then then items $(i)-(i v)$ from Lemma 5.2 hold, and in addition,

(v) $E_{x y}=0$ if $x, y \in V(G)$ or $x, y \in V(H)$;

(vi) $E_{x y}=E_{y x}$ for all $x, y \in V$.

Proof. The first four conditions follow immediately from Lemma 5.2. For $(v)$, consider $g, g^{\prime} \in V(G)$ and note that

$$
0=\sum_{y \in V} p\left(g^{\prime}, y \mid g, x\right)=\sum_{y \in V} \psi^{\dagger}\left(E_{g g^{\prime}} \otimes F_{x y}\right) \psi=\psi^{\dagger}\left(E_{g g^{\prime}} \otimes I\right) \psi=\operatorname{Tr}\left(\rho \rho^{\dagger} E_{g g^{\prime}}\right),
$$

where the last equality follows from Equation (17) and the cyclicity of the trace. Since we are working in the Schmidt basis of $\psi$, the matrix $\rho$ is diagonal with strictly positive diagonal entries. Therefore $\rho \rho^{\dagger}$ has full rank and thus it follows by (21) that $E_{g g^{\prime}}=0$. Similarly, we have that $E_{h h^{\prime}}=0$ for all $h, h^{\prime} \in V(H)$.

Lastly, we show $(v i)$. By the rules of the $(G, H)$-isomorphism game, we must have that $p\left(y, x^{\prime} \mid x, y\right)=0$ whenever $x^{\prime} \neq x$. Thus by $(i v)$ we have that $E_{x y} E_{y x^{\prime}}=0$ for all $x^{\prime} \neq x$. Therefore,

$$
E_{x y}=E_{x y} \sum_{x^{\prime}} E_{y x^{\prime}}=E_{x y} E_{y x}
$$

Also, $E_{x y^{\prime}} E_{y x}=0$ for $y^{\prime} \neq y$, and thus

$$
E_{y x}=\sum_{y^{\prime}} E_{x y^{\prime}} E_{y x}=E_{x y} E_{y x}
$$

Combining the two equations above we get that $E_{x y}=E_{y x}$.

\subsection{Two algebraic reformulations}

In this section we use the structural properties of perfect quantum strategies to the $(G, H)$ isomorphism game we identified in Section 5.2 to prove Result 2 .

First, we show the equivalence $(i) \Longleftrightarrow(i i)$ from Result 2 . 
Theorem 5.4. Let $G$ and $H$ be graphs. Then $G \cong_{q} H$ if and only if there exist projectors $E_{g h}$ for $g \in V(G)$ and $h \in V(H)$ such that

(i) $\sum_{h \in V(H)} E_{g h}=I$, for all $g \in V(G)$;

(ii) $\sum_{g \in V(G)} E_{g h}=I$, for all $h \in V(H)$;

(iii) $E_{g h} E_{g^{\prime} h^{\prime}}=0$, if $\operatorname{rel}\left(g, g^{\prime}\right) \neq \operatorname{rel}\left(h, h^{\prime}\right)$.

Proof. Using Theorem 5.3, it is relatively easy to see that Alice's operators $E_{g h}$ for $g \in V(G)$, $h \in V(H)$ from a perfect quantum strategy satisfy Conditions $(i)$ (ii) and (iii).

Conversely, suppose that $E_{g h}$ for $g \in V(G)$ and $h \in V(H)$ satisfy the hypotheses of the theorem. Define $E_{h g}=E_{g h}$, and $E_{g g^{\prime}}=E_{h h^{\prime}}=0$ for all $h, h^{\prime} \in V(H), g, g^{\prime} \in V(G)$. Furthermore, let $F_{x y}=E_{x y}^{\top}$ for all $x, y \in V(G) \cup V(H)$. It is easy to see that $\mathcal{E}_{x}=\left\{E_{x y}: y \in V(G) \cup V(H)\right\}$ is a valid measurement for all $x \in V(G) \cup V(H)$, and similarly for $\mathcal{F}_{x}=\left\{F_{x y}: y \in V(G) \cup V(H)\right\}$. Consider the quantum strategy where Alice and Bob respectively use the measurements $\mathcal{E}_{x}$ and $\mathcal{F}_{x}$ on a shared maximally entangled state $\psi_{d}=\frac{1}{\sqrt{d}} \sum_{i=1}^{d} e_{i} \otimes e_{i}$. By (3) we have that

$$
p\left(y, y^{\prime} \mid x, x^{\prime}\right)=\psi_{d}^{\dagger}\left(E_{x y} \otimes F_{x^{\prime} y^{\prime}}\right) \psi_{d}=\frac{1}{d} \operatorname{Tr}\left(E_{x y} E_{x^{\prime} y^{\prime}}\right),
$$

for all $x, x^{\prime}, y, y^{\prime} \in V(G) \cup V(H)$. This fact combined with Condition (iii) shows that this is a perfect strategy for the $(G, H)$-isomorphism game.

Recall that an alternative characterization of graph isomorphism is given by the equation $A_{G} P=$ $P A_{H}$ for some permutation matrix $P$, where $A_{G}$ and $A_{H}$ are the adjacency matrices of two graphs. It turns out that one can use Theorem 5.4 to obtain an analogous formulation of quantum graph isomorphism. First we will need the following definition:

Definition 5.5. A matrix $\mathcal{P} \in \mathbb{M}_{n}(d)$ is called a projective permutation matrix of block size $d$ if it is unitary and all of its blocks are projectors.

Note that a projective permutation matrix of block size one is a unitary matrix whose entries square to themselves, i.e., a permutation matrix. The following lemma shows that projective permutation matrices can be built out of projectors satisfying the first two conditions of Theorem 5.4 .

Lemma 5.6. A matrix $\mathcal{P}=\left[\left[E_{i j}\right]\right] \in \mathbb{M}_{n}(d)$ is a projective permutation matrix if and only if the matrix $E_{i j}$ is a projector for all $i, j \in[n]$ and

(i) $\sum_{j=1}^{n} E_{i j}=I$, for all $i \in[n]$;

(ii) $\sum_{i=1}^{n} E_{i j}=I$, for all $j \in[n]$.

Proof. It suffices that show that, assuming the matrices $\left\{E_{i j}\right\}_{i j}$ are projectors, Conditions $(i)$ and $(i i)$ in the statement of the lemma are equivalent to $\mathcal{P}$ being unitary.

First, assume that Conditions $(i)$ and $(i i)$ hold. Since the matrices $\left\{E_{i j}\right\}_{i j}$ are projectors it follows that $E_{i k} E_{j k}=0$, for all $i \neq j \in[n]$. This implies

$$
\left(\mathcal{P} \mathcal{P}^{\dagger}\right)_{i, j}=\sum_{k} E_{i k} E_{j k}= \begin{cases}\sum_{k} E_{i k}^{2}=I, & \text { if } i=j \\ 0, & \text { if } i \neq j\end{cases}
$$


Therefore $\mathcal{P} \mathcal{P}^{\dagger}=I$ and similarly we have that $\mathcal{P}^{\dagger} \mathcal{P}=I$, i.e., $\mathcal{P}$ is unitary.

Conversely, suppose that $\mathcal{P}$ is unitary. Since $\mathcal{P} \mathcal{P}^{\dagger}=I$ we have that

$$
I=\left(\mathcal{P} \mathcal{P}^{\dagger}\right)_{i, i}=\sum_{j} E_{i j}^{2}=\sum_{j} E_{i j}
$$

Analogously, using that $\mathcal{P}^{\dagger} \mathcal{P}=I$, we get that $\sum_{i} E_{i j}=I$, and thus Conditions $(i)$ and $(i i)$ hold.

Remark 5.7. In [21], Musto and Vicary introduced quantum Latin squares. This is an $n \times n$ array of unit vectors in which each row and column forms an orthonormal basis of $\mathbb{C}^{n}$. They use quantum Latin squares to construct unitary error bases which are related to teleportation, dense coding, and quantum error correction. If $\mathcal{P}$ is a projective permutation matrix in which each projector $E_{i j}$ has rank one, then there exist unit vectors $\psi_{i j}$ such that $E_{i j}=\psi_{i j} \psi_{i j}^{\dagger}$. By Lemma 5.6 we have that $\psi_{i j}^{\dagger} \psi_{i j^{\prime}}=0$ when $j \neq j^{\prime}$ and $\psi_{i j}^{\dagger} \psi_{i^{\prime} j}=0$ when $i \neq i^{\prime}$. In other words, the vectors $\psi_{i j}$ form a quantum Latin square. Thus projective permutation matrices also generalize quantum Latin squares.

Next, we prove a useful lemma:

Lemma 5.8. Consider two graphs $G$ and $H$, and let $\mathcal{P}=\left[\left[E_{g h}\right]\right] \in \mathbb{M}_{n}(d)$ (for some $d \in \mathbb{N}$ ) be a projective permutation matrix. Then, $\mathcal{P}$ satisfies

$$
\left(A_{G} \otimes I_{d}\right) \mathcal{P}=\mathcal{P}\left(A_{H} \otimes I_{d}\right),
$$

if and only if, it satisfies

$$
E_{g h} E_{g^{\prime} h^{\prime}}=0, \text { when } \operatorname{rel}\left(g, g^{\prime}\right) \neq \operatorname{rel}\left(h, h^{\prime}\right)
$$

Proof. As $\mathcal{P}$ is a projective permutation matrix, note that the conditions $E_{g h} E_{g^{\prime} h^{\prime}}=0$ whenever $\left(g=g^{\prime}\right.$ and $\left.h \neq h^{\prime}\right)$ or $\left(h=h^{\prime}\right.$ and $\left.g \neq g^{\prime}\right)$ are already guaranteed by Conditions $(i)$ and $(i i)$ of Lemma 5.6. Thus, we only need to prove the remaining orthogonalities in (23).

First, we assume that (23) holds. The $\left(g, g^{\prime}\right)$-block of $A_{G} \otimes I_{d}$ is equal to $I_{d}$ if $g$ and $g^{\prime}$ are adjacent, and is 0 otherwise. Similarly for the $\left(h, h^{\prime}\right)$-block of $A_{H} \otimes I_{d}$. Moreover, note that for $g \in V(G)$ and $h \in V(H)$ we have

$$
\left(\left(A_{G} \otimes I_{d}\right) \mathcal{P}\right)_{g, h}=\sum_{g^{\prime}: g^{\prime} \sim g} E_{g^{\prime} h}, \quad \text { and } \quad\left(\mathcal{P}\left(A_{H} \otimes I_{d}\right)\right)_{g, h}=\sum_{h^{\prime}: h^{\prime} \sim h} E_{g h^{\prime}}
$$

Using (23), for all $g \in V(G)$ and $h \in V(H)$ we have

$$
\sum_{g^{\prime}: g^{\prime} \sim g} E_{g^{\prime} h}=\sum_{g^{\prime}: g^{\prime} \sim g} E_{g^{\prime} h} \sum_{h^{\prime}} E_{g h^{\prime}}=\sum_{g^{\prime}: g^{\prime} \sim g} E_{g^{\prime} h} \sum_{h^{\prime}: h^{\prime} \sim h} E_{g h^{\prime}}=\sum_{g^{\prime}} E_{g^{\prime} h} \sum_{h^{\prime}: h^{\prime} \sim h} E_{g h^{\prime}}=\sum_{h^{\prime}: h^{\prime} \sim h} E_{g h^{\prime}},
$$

and therefore, using 24 it follows that $\left(A_{G} \otimes I_{d}\right) \mathcal{P}=\mathcal{P}\left(A_{H} \otimes I_{d}\right)$.

Conversely, if $\left(A_{G} \otimes I_{d}\right) \mathcal{P}=\mathcal{P}\left(A_{H} \otimes I_{d}\right)$, it follows by (24) that

$$
\sum_{g^{\prime}: g^{\prime} \sim g} E_{g^{\prime} h}=\sum_{h^{\prime}: h^{\prime} \sim h} E_{g h^{\prime}}, \text { for all } g \in V(G), h \in V(H) .
$$


Furthermore, since the projectors $E_{g h}$ are mutually orthogonal for any fixed $h \in V(H)$ we have

$$
\left(\sum_{g^{\prime}: g^{\prime} \sim g} E_{g^{\prime} h}\right)^{2}=\sum_{g^{\prime}: g^{\prime} \sim g} E_{g^{\prime} h},
$$

and therefore, combining (25) with 26 it follows that

$$
\sum_{g^{\prime} \sim g} E_{g^{\prime} h} \sum_{h^{\prime} \sim h} E_{g h^{\prime}}=\sum_{g^{\prime} \sim g} E_{g^{\prime} h}=\sum_{g^{\prime} \sim g} E_{g^{\prime} h} \sum_{h^{\prime}} E_{g h^{\prime}} .
$$

As a consequence of (27) we get that

$$
\sum_{g^{\prime} \sim g} E_{g^{\prime} h} \sum_{h^{\prime} \nsim h} E_{g h^{\prime}}=0
$$

Taking traces in 28$)$ we have

$$
\sum_{g^{\prime}: g^{\prime} \sim g, h^{\prime}: h^{\prime} \nsim h} \operatorname{Tr}\left(E_{g^{\prime} h} E_{g h^{\prime}}\right)=0 .
$$

Since the matrices $E_{g h}$ are positive semidefinite (as they are projectors), all the terms in (29) must be nonnegative. Therefore, we have that $\operatorname{Tr}\left(E_{g^{\prime} h} E_{g h^{\prime}}\right)=0$ for all $g^{\prime} \sim g$ and $h^{\prime} \not h$, which implies that $E_{g^{\prime} h} E_{g h^{\prime}}=0$ for all $g^{\prime} \sim g$ and $h^{\prime} \not h$. One can similarly show that $E_{g^{\prime} h} E_{g h^{\prime}}=0$ if $h \sim h^{\prime}$ and $g \nsim g^{\prime}$. So if one of $\operatorname{rel}\left(g, g^{\prime}\right)$ and $\operatorname{rel}\left(h, h^{\prime}\right)$ is "adjacency" and the other is not, we have the desired orthogonalities. We also already noted at the beginning of the proof that when one of $\operatorname{rel}\left(g, g^{\prime}\right)$ and $\operatorname{rel}\left(h, h^{\prime}\right)$ is "equality" and the other is not, we have the required orthogonality. The only remaining case is when one of $\operatorname{rel}\left(g, g^{\prime}\right)$ and $\operatorname{rel}\left(h, h^{\prime}\right)$ is "distinct non-adjacency" and the other is not. However this is implied by what we already have, since the relationship which is not "distinct non-adjacency" will be one of "equality" or "adjacency".

We are now ready to prove the equivalence $(i) \Longleftrightarrow($ iii $)$ from Result 2 ,

Theorem 5.9. For any two graphs $G$ and $H$ we have that $G \cong_{q} H$ if and only if there exists a projective permutation matrix $\mathcal{P} \in \mathbb{M}_{n}(d)$ (for some $d \in \mathbb{N}$ ) such that

$$
\left(A_{G} \otimes I_{d}\right) \mathcal{P}=\mathcal{P}\left(A_{H} \otimes I_{d}\right) .
$$

Proof. First, assume that $G \cong_{q} H$. By Theorem 5.4, there exists a projective permutation matrix $\mathcal{P}=\left[\left[E_{g h}\right]\right] \in \mathbb{M}_{n}(d)$ (for some $d \in \mathbb{N}$ ) satisfying (23). Thus, by Lemma 5.8, $\mathcal{P}$ also satisfies $\left(A_{G} \otimes I_{d}\right) \mathcal{P}=\mathcal{P}\left(A_{H} \otimes I_{d}\right)$. Conversely, let $\mathcal{P}=\left[\left[E_{g h}\right]\right] \in \mathbb{M}_{n}(d)$ (for some $d \in \mathbb{N}$ ) be a projective permutation matrix satisfying $\left(A_{G} \otimes I_{d}\right) \mathcal{P}=\mathcal{P}\left(A_{H} \otimes I_{d}\right)$. Then, by Lemma 5.8, $\mathcal{P}$ satisfies (23). Lastly, by Theorem 5.4 we get that $G \cong_{q} H$.

Remark 5.10. Theorem 5.9 shows that projective permutation matrices play the role of permutation matrices for quantum isomorphisms. In fact, just as any permutation matrix corresponds to an isomorphism from a complete (or empty) graph to itself, any projective permutation matrix corresponds to a quantum isomorphism from a complete (or empty) graph to itself. 
Since a projective permutation matrix is unitary, the equation $\left(A_{G} \otimes I_{d}\right) \mathcal{P}=\mathcal{P}\left(A_{H} \otimes I_{d}\right)$ is equivalent to $\mathcal{P}^{\dagger}\left(A_{G} \otimes I_{d}\right) \mathcal{P}=\left(A_{H} \otimes I_{d}\right)$, which implies that $A_{G} \otimes I_{d}$ and $A_{H} \otimes I_{d}$ have the same multiset of eigenvalues. Of course this means that $A_{G}$ and $A_{H}$ have the same multiset of eigenvalues, and thus quantum isomorphic graphs are cospectral with respect to their adjacency matrices. Since two graphs are quantum isomorphic if and only if their complements are, we have the following corollary:

Corollary 5.11. If $G \cong_{q} H$ then $G$ and $H$ are cospectral with cospectral complements.

Note that this is not the case for non-signalling/fractional isomorphism. Indeed, any two $n$ vertex, $k$-regular graphs are fractionally isomorphic but there are many such pairs that are not cospectral. From this it follows that quantum and non-signalling isomorphism are different:

Corollary 5.12. There exist graphs that are non-signalling isomorphic but not quantum isomorphic.

In [15] we show that cospectrality is a consequence of a semidefinite relaxation of quantum isomorphism that we call $\mathcal{S}_{+}$-isomorphism. This relation is strictly weaker than quantum isomorphism, but still stronger than non-signalling isomorphism.

\subsection{Quantum commuting isomorphisms}

So far we have discussed the finite dimensional tensor product framework for quantum mechanics and thus for playing nonlocal games. However, other models exist: as one example, one can consider allowing infinite dimensional Hilbert spaces to model the quantum systems of the players. In this case, the strategies are the same and the probabilities are given by the same expression, but the shared state $\psi$ and the operators $E_{x y}$ and $F_{x y}$ for Alice and Bob are allowed to be infinite dimensional. In general, it is not known whether allowing infinite dimensional spaces can allow one to win a nonlocal game perfectly when one cannot using finite dimensional strategies. However, though it is not obvious, it follows from [11] that these two models for quantum strategies are equivalent for the isomorphism game.

In this section we focus on the quantum commuting framework, where the state of the shared system is described by a unit vector in a potentially infinite-dimensional space $H$, and the meausurement operators correspond to positive bounded operators acting on $H$, such that Alice's measurement operators commute with all of Bob's measurement operators. In this setting, two graphs $G$ and $H$ are quantum commuting isomorphic, denoted $G \cong_{q c} H$, whenever there exists a perfect quantum commuting strategy for the $(G, H)$-isomorphism game.

Quantum commuting strategies were investigated in [26] and [25], for the nonlocal game corresponding to graph coloring. Nevertheless, the analysis from [25] extends to any synchronous game, and using their results we can obtain a characterization for the existence of perfect quantum commuting strategies for any synchronous game. Before we give the formal statement we need the following definitions.

A tracial state on a unital $C^{*}$-algebra $\mathcal{A}$ is a linear functional $s: \mathcal{A} \rightarrow \mathbb{C}$ such that $s(I)=1$, $s\left(A^{*} A\right) \geq 0$ for all $A \in \mathcal{A}$, and $s(A B)=s(B A)$ for all $A, B \in \mathcal{A}$. The tracial state $s$ is faithful if $s\left(A^{*} A\right)=0$ if and only if $A=0$. Note that if $A$ and $B$ are projections, then $s(A B)=0$ implies that $A B=0$ just like in the finite dimensional case (as long as $s$ is faithful). For the finite dimensional matrix algebra $\mathbb{C}^{d \times d}$, there is a unique tracial state given by $s(M)=\operatorname{Tr}(M) / d$. 
Lemma 5.13. Consider a synchronous game with input sets $X$, output sets $Y$, and Boolean predicate $V$. There exists a perfect quantum commuting strategy for this game if and only if there exists a unital $C^{*}$-algebra $\mathcal{A}$, a faithful tracial state $s: \mathcal{A} \rightarrow \mathbb{C}$, and projections $E_{x y} \in \mathcal{A}$ for $(x, y) \in X \times Y$ such that

(i) $\sum_{y \in Y} E_{x y}=I$;

(ii) $s\left(E_{x y} E_{x^{\prime} y^{\prime}}\right)=0$ if $V\left(y, y^{\prime} \mid x, x^{\prime}\right)=0$.

Using Lemma 5.13, we can prove the following analog of Theorem 5.4. We omit the proof since it is similar to that of Theorem 5.4 .

Theorem 5.14. Let $G$ and $H$ be graphs. Then $G \cong_{q c} H$ if and only if there exists a $C^{*}$-algebra $\mathcal{A}$ which admits a faithful tracial state, and projections $E_{g h} \in \mathcal{A}$ for $g \in V(G)$ and $h \in V(H)$ such that

(i) $\sum_{h \in V(H)} E_{g h}=I$, for all $g \in V(G)$;

(ii) $\sum_{g \in V(G)} E_{g h}=I$, for all $h \in V(H)$;

(iii) $E_{g h} E_{g^{\prime} h^{\prime}}=0$, if $\operatorname{rel}\left(g, g^{\prime}\right) \neq \operatorname{rel}\left(h, h^{\prime}\right)$.

Note that since quantum commuting strategies for nonlocal games are more general than quantum tensor product strategies, we have that two graphs being quantum isomorphic implies that they are also quantum commuting isomorphic. Similarly, since quantum commuting strategies are also non-signalling, we have that any two quantum commuting isomorphic graphs are non-signalling isomorphic. In summary, we have that for any two graphs $G$ and $H$

$$
G \cong H \Rightarrow G \cong_{q} H \Rightarrow G \cong_{q c} H \Rightarrow G \cong_{n s} H .
$$

By the end of this work we will see that all of these implications are strict, i.e., none of the four relations above are equivalent.

\subsection{Necessary conditions from the existence of quantum homomorphisms}

In this section we give necessary conditions for a pair of graphs to be quantum isomorphic, in terms of homomorphisms. A graph homomorphism from $G$ to $H$ is an adjacency preserving function $\varphi: V(G) \rightarrow V(H)$, i.e., if $g \sim g^{\prime}$ then $\varphi(g) \sim \varphi\left(g^{\prime}\right)$. When such a function exists, we write $G \rightarrow H$.

Graph homomorphisms provide a framework which unifies many aspects of graph theory. In particular, many graph parameters can be defined in terms of graph homomorphisms. We will be primarily concerned with three such parameters. The chromatic number of a graph $G$, denoted $\chi(G)$, is the minimum number of colors required to color the vertices of $G$ such that no two adjacent vertices are assigned the same color. Equivalently, the chromatic number of $G$ is the size of the smallest complete graph to which $G$ admits a homomorphism.=, i.e., $\chi(G)=\min \left\{c: G \rightarrow K_{c}\right\}$, where $K_{c}$ is the complete graph on $c$ vertices. The clique number of $G$, denoted $\omega(G)$, is the size of the largest set of pairwise adjacent vertices in $G$. Equivalently, $\omega(G)=\max \left\{c: K_{c} \rightarrow G\right\}$. The independence number of $G$, denoted $\alpha(G)$, is the maximum size of a set of pairwise non-adjacent vertices of $G$. Obviously, $\alpha(G)=\omega(\bar{G})$. Below, we will introduce some quantum analogs of these parameters using the notion of quantum homomorphisms. 
Given graphs $G$ and $H$, the $(G, H)$-homomorphism game is played as follows: Alice and Bob are given vertices $g_{A}, g_{B} \in V(G)$ and must respond with vertices $h_{A}, h_{B} \in V(H)$ respectively. If $g_{A}=g_{B}$, then to win they must satisfy $h_{A}=h_{B}$, and if $g_{A} \sim g_{B}$ they must satisfy $h_{A} \sim h_{B}$. Similarly to the isomorphism game, it is not difficult to show that classical players can win the $(G, H)$-homomorphism game perfectly if and only if there exists a homomorphism from $G$ to $H$. Motivated by this, in [16] the authors say that there is a quantum homomorphism from $G$ to $H$, and write $G \stackrel{q}{\rightarrow} H$, if there exists a perfect quantum strategy for the $(G, H)$-homomorphism game.

Lemma 5.15. If $G \cong_{q} H$, we have that $G \stackrel{q}{\rightarrow} H, H \stackrel{q}{\rightarrow} G, \bar{G} \stackrel{q}{\rightarrow} \bar{H}$, and $\bar{H} \stackrel{q}{\rightarrow} \bar{G}$.

Proof. Suppose that Alice and Bob have a perfect strategy for the $(G, H)$-isomorphism game. If we restrict their possible inputs to only the vertices from $G$, then it is easy to see that they will always satisfy the winning conditions of the $(G, H)$-homomorphism game: if they are given the same vertices from $G$ they will respond with the same vertices from $H$, and if they are given adjacent vertices of $G$ they will respond with adjacent vertices of $H$. Therefore, if two players have a perfect (classical or quantum) strategy for the $(G, H)$-isomorphism game, they can use the same strategy, but restricted to inputs from $V(G)$, to perfectly win the $(G, H)$-homomorphism game.

Lemma 5.15 is useful as it can be combined with known results relating quantum homomorphisms and certain quantum analogs of various classical graph parameters defined below.

Definition 5.16. The quantum chromatic number of $G$, denoted $\chi_{q}(G)$, is defined as the minimum $c \in \mathbb{N}$ such that $G \stackrel{q}{\rightarrow} K_{c}$. The quantum clique number of $G$, denoted $\omega_{q}(G)$, is the minimum $c \in \mathbb{N}$ such that $K_{c} \stackrel{q}{\rightarrow} G$. Lastly, the quantum independence number of $G$ is defined as $\alpha_{q}(G):=\omega_{q}(\bar{G})$.

It follows essentially from the definition that $\chi_{q}$ is quantum homomorphism monotone, i.e., that $G \stackrel{q}{\rightarrow} H$ implies $\chi_{q}(G) \leq \chi_{q}(H)$ [16. By Lemma 5.15, this shows that $G \cong_{q} H$ implies $\chi_{q}(G)=\chi_{q}(H)$, and similarly for their complements. Similarly to $\chi_{q}$, the parameters $\alpha_{q}$ and $\omega_{q}$ are also equal for a pair of quantum isomorphic graphs.

For the quantum graph parameters introduced above, proving quantum homomorphism monotonicity is straightforward, since the parameters themselves are defined in terms of quantum homomorphisms. However, there are some interesting examples of graph parameters that are not defined in this way, but still turn out to be quantum homomorphism monotone. For instance, the well known Lovász theta number (of the complement) was proven to be quantum homomorphism monotone in [16], as were two variants by Schrijver and Szegedy in [29]. For us, there are two other, lesser known, quantum homomorphism monotone parameters that will be important for this work.

A projective packing of a graph $G$ is an assignment, $g \mapsto E_{g} \in \mathbb{C}^{d \times d}$, of $d \times d$ projectors for some $d \in \mathbb{N}$ such that adjacent vertices receive orthogonal projectors. Note that there is no uniformity condition on the rank of the projectors as there is for a projective representation. The value of a projective packing is equal to $\frac{1}{d} \sum_{g \in V(G)} \mathrm{rk}\left(E_{g}\right)$, and the projective packing number of $G$, denoted $\alpha_{p}(G)$, is the supremum of the values of all projective packings of $G$.

Remark 5.17. Like the quantum independence number, the projective packing number is meant to be analogous to, but a relaxation of, the usual independence number of a graph. Indeed, if $S \subseteq V(G)$ is an independent set in $G$, then assigning identity to every element of $S$ and assigning zero to every other vertex produces a projective packing of value $|S|$. It follows that $\alpha(G) \leq \alpha_{p}(G)$. 
It is further known that $\alpha_{q}(G) \leq \alpha_{p}(G)$ [29]. In fact, there is no graph $G$ for which it is known that $\alpha_{q}(G) \neq\left\lfloor\alpha_{p}(G)\right\rfloor$.

Lemma 5.18. If $G \cong_{q} H$ then $\alpha_{p}(G)=\alpha_{p}(H)$ and $\alpha_{p}(\bar{G})=\alpha_{p}(\bar{H})$. Moreover, if $G$ has $a$ projective packing of value $\gamma$ then $H$ has a projective packing of value $\gamma$.

Proof. It was shown in [29] that $G \stackrel{q}{\rightarrow} H$ implies that $\alpha_{p}(\bar{G}) \leq \alpha_{p}(\bar{H})$, and moreover if $\bar{G}$ has a projective packing of value $\gamma$ then $\bar{H}$ has a projective packing of value $\gamma$. As $G \cong_{q} H$, by Lemma 5.15 we get that $G \stackrel{q}{\rightarrow} H, H \stackrel{q}{\rightarrow} G$, and $\bar{G} \stackrel{q}{\rightarrow} \bar{H}, \bar{H} \stackrel{q}{\rightarrow} \bar{G}$ which imply that $\alpha_{p}(\bar{G})=\alpha_{p}(\bar{H})$, and $\alpha_{p}(G)=\alpha_{p}(H)$, respectively.

In this work we will use Lemma 5.18 for our reduction of linear binary constraint system games to isomorphism games in Section 6.2. We will also make use of the following which was shown in [17]:

Lemma 5.19. Let $G$ be a graph. Then $\alpha_{p}(G) \leq \chi(\bar{G})$, and there exists a projective packing of $G$ of value $\chi(\bar{G})$ if and only if $\alpha_{q}(G)=\chi(\bar{G})$.

A $(d / r)$-projective representation of a graph $G$ is a projective packing of $d \times d$ projectors with the additional constraint that every projector has rank $r$. The projective rank of a graph $G$, denoted by $\xi_{f}(G)$, is the infimum of $\frac{d}{r}$ such that $G$ has a $(d / r)$-projective representation.

Lemma 5.20. If $G \cong_{q} H$ then $\xi_{f}(G)=\xi_{f}(H)$ and $\xi_{f}(\bar{G})=\xi_{f}(\bar{H})$. Moreover, if $G$ has a $(d / r)$ projective representation then $H$ has a $\left(d^{\prime} / r^{\prime}\right)$-projective representation where $\frac{d^{\prime}}{r^{\prime}}=\frac{d}{r}$.

Proof. It was shown in [16] that $\xi_{f}$ is quantum homomorphism monotone, and moreover, if $G \stackrel{q}{\rightarrow} H$ and $H$ has a $(d / r)$-projective representation then $G$ has a $\left(d^{\prime} / r^{\prime}\right)$-projective representation for some $d^{\prime}, r^{\prime}$ such that $\frac{d^{\prime}}{r^{\prime}}=\frac{d}{r}$. As $G \cong_{q} H$, by Lemma 5.15 we have that $G \stackrel{q}{\rightarrow} H$ and $H \stackrel{q}{\rightarrow} G$, and thus, $\xi_{f}(G)=\xi_{f}(H)$. Similarly, we get that $\xi_{f}(\bar{G})=\overline{\xi_{f}(H)}$.

In our upcoming work [18, we use Lemma 5.20 above to show that quantum isomorphism and one of our semidefinite relaxations of quantum isomorphism are indeed different relations.

\section{Separating isomorphism and quantum isomorphism}

In this section we prove Result 3, i.e., we construct pairs of graphs that are quantum isomorphic but not isomorphic. For this we introduce a type of game investigated by Cleve and Mittal [11] known as binary constraint system (BCS) games. We will show that, in the linear case, one can reduce the existence of a perfect classical (quantum) strategy for a BCS game to the existence of a perfect classical (quantum) strategy to a corresponding isomorphism game.

\subsection{Binary constraint systems games}

A linear binary constraint system (BCS) $\mathcal{F}$ consists of a family of binary variables $x_{1}, \ldots, x_{n}$ and constraints $C_{1}, \ldots, C_{m}$, where each $C_{\ell}$ is a linear equation over $\mathbb{F}_{2}$ in some subset of the variables. Thus $C_{\ell}$ takes the form $\sum_{x_{i} \in S_{\ell}} x_{i}=b_{\ell}$ for some $S_{\ell} \subseteq\left\{x_{1}, \ldots, x_{n}\right\}$ and $b_{\ell} \in\{0,1\}$. We say that a BCS is satisfiable if there is an assignment of values from $\mathbb{F}_{2}$ to the variables $x_{i}$ such that every constraint $C_{\ell}$ is satisfied. Such an assignment is known as a satisfying assignment. 
An example of a linear BCS is the following:

$$
\begin{array}{ll}
x_{1}+x_{2}+x_{3}=0 & x_{1}+x_{4}+x_{7}=0 \\
x_{4}+x_{5}+x_{6}=0 & x_{2}+x_{5}+x_{8}=0 \\
x_{7}+x_{8}+x_{9}=0 & x_{3}+x_{6}+x_{9}=1
\end{array}
$$

where addition is over $\mathbb{F}_{2}$. Note that the BCS given above is not satisfiable. Indeed, every variable appears in exactly two constraints and thus summing up all equations modulo 2 we get $0=1$.

To any linear BCS $\mathcal{F}$ we associate the following nonlocal game, which we call the $B C S$ game. In the BCS game, the verifier gives Alice a constraint $C_{\ell}$ and Bob a constraint $C_{k}$. In order to win, they must each respond with an assignment of values to the variables in their respective constraints such that those constraints are satisfied. Furthermore, for the variables in $S_{\ell} \cap S_{k}$, Alice and Bob

must agree on their assignment. Note that if they are given the same constraint, these conditions imply that they must give the same response.

It is easy to see that Alice and Bob can win the BCS game classically with probability 1 if and only if the corresponding BCS is satisfiable. This motivates the following definition.

Definition 6.1. A linear BCS is called quantum satisfiable if there exists a perfect quantum strategy for the corresponding BCS game.

We note that Cleve and Mittal in [1] also define a nonlocal game corresponding to a linear BCS that admits a perfect classical strategy if and only if the underlying BCS is satisfiable. However, their construction is slightly different than the game we devise here. Specifically, in their game, the verifier gives Alice a constraint $C_{\ell}$ and to Bob a single variable $x_{i} \in C_{\ell}$. Alice returns an assignment for the variables in her constraint and Bob an assignment for his variable. The winning conditions are that $(i)$ Alice's assignment must satisfy $C_{\ell}$ and (ii) Bob's assignment on $x_{i}$ must be consistent with Alice's assignment. Moreover, they define quantum satisfiability in terms of something they call a quantum satisfying assignment for the equations in the BCS. However, their main result is that a BCS game has a perfect quantum strategy if and only if the BCS has a quantum satisfying assignment, and from this it easily follows that a perfect quantum strategy exists for our version of the BCS game if and only if one exists for theirs. Moreover, this implies that our notion of quantum satisfiability is the same as theirs. In the case of quantum commuting strategies, the equivalence of the two versions of the BCS game follow from the results in [10]. The reason we define the game differently is simply because the reduction to quantum isomorphism is more natural for this version.

There are many classes of linear BCS's that are quantum satisfiable but not satisfiable. Indeed, the example given above in (32) corresponds to the Mermin-Peres magic square game [20] which has a perfect quantum strategy. One can also use a result of Arkhipov [1] to construct a linear BCS that is quantum satisfiable but not satisfiable from any non-planar graph.

\subsection{The Reduction}

In this section we prove that (quantum) satisfiability of a linear BCS can be reduced to (quantum) graph isomorphism. As a first step we introduce the graphs we use in the reduction.

To any linear BCS $\mathcal{F}$ with $m$ constraints we associate the graph $G_{\mathcal{F}}$ which is defined as follows: For each constraint $C_{\ell}$, and each assignment $f: S_{\ell} \rightarrow \mathbb{F}_{2}$ that satisfies $C_{\ell}$ we include a vertex $(\ell, f)$. Furthermore, we add an edge between two vertices $(\ell, f)$ and $\left(k, f^{\prime}\right)$ if they are inconsistent, i.e., 
if there exists $x_{i} \in S_{\ell} \cap S_{k}$ such that $f\left(x_{i}\right) \neq f^{\prime}\left(x_{i}\right)$. We remark that this construction is related to the FGLSS reduction from [12, which is well known in the approximability literature. The construction is also closely connected to the CFI graphs [8, whose construction in terms of linear systems can be found in [7].

Note that all vertices of $G_{\mathcal{F}}$ corresponding to a fixed constraint are pairwise adjacent. Thus, for any linear BCS $\mathcal{F}$, any independent set in $G_{\mathcal{F}}$ contains at most one vertex corresponding to each constraint. Therefore $\alpha\left(G_{\mathcal{F}}\right) \leq m$ for any linear BCS $\mathcal{F}$ with $m$ constraints.

Given any linear BCS $\mathcal{F}$, we define the homogenization of $\mathcal{F}$, denoted by $\mathcal{F}_{0}$, to be the linear BCS obtained from $\mathcal{F}$ by changing the righthand sides of all of the constraints to 0 . Note that the homogenization of a linear BCS always has a solution, namely the all-zero assignment. Also note that $G_{\mathcal{F}}$ and $G_{\mathcal{F}_{0}}$ have the same number of vertices.

We now show that $G_{\mathcal{F}_{0}}$ always contains an independent set of size $m$ (and thus $\alpha\left(G_{\mathcal{F}_{0}}\right)=m$ ). For each constraint $C_{\ell}$, let $f_{\ell}^{0}$ denote the zero assignment to the variables in $S_{\ell}$. Note that $\left(\ell, f_{\ell}^{0}\right)$ is a vertex of $G_{\mathcal{F}_{0}}$. Moreover, $\left(\ell, f_{\ell}^{0}\right)$ and $\left(k, f_{k}^{0}\right)$ are not adjacent in $G_{\mathcal{F}_{0}}$ since $f_{\ell}^{0}$ and $f_{k}^{0}$ are just restrictions of the same function (the zero assignment to all variables) and thus they necessarily agree on the intersection of their domains. Thus the vertices $\left\{\left(\ell, f_{\ell}^{0}\right): \ell \in[m]\right\}$ form an independent set in $G_{\mathcal{F}_{0}}$ of size $m$. Therefore, for any linear BCS $\mathcal{F}$ with $m$ constraints we have

$$
\alpha\left(G_{\mathcal{F}_{0}}\right)=m .
$$

We are now ready to prove that satisfiability of a linear BCS $\mathcal{F}$ can be reduced to deciding whether $G_{\mathcal{F}}$ and $G_{\mathcal{F}_{0}}$ are isomorphic.

Theorem 6.2. Let $\mathcal{F}$ be a linear BCS with $m$ constraints. Then the following are equivalent:

(i) $\mathcal{F}$ is satisfiable;

(ii) The graphs $G_{\mathcal{F}}$ and $G_{\mathcal{F}_{0}}$ are isomorphic;

(iii) $\alpha\left(G_{\mathcal{F}}\right)=m$.

Proof. $(i) \Longrightarrow($ ii $)$. Suppose that $\mathcal{F}$ is satisfiable and let $F:\left\{x_{1}, \ldots, x_{n}\right\} \rightarrow \mathbb{F}_{2}$ be a satisfying assignment. For each constraint $C_{\ell}$, let $F_{\ell}$ be the restriction of $F$ to the set $S_{\ell}$. Define a function $\varphi: V\left(G_{\mathcal{F}}\right) \rightarrow V\left(G_{\mathcal{F}_{0}}\right)$ as follows. For each vertex $(\ell, f)$ of $G_{\mathcal{F}}$, set $\varphi(\ell, f)=\left(\ell, f \oplus F_{\ell}\right)$, where $f \oplus F_{\ell}$ is defined to be the function from $S_{\ell}$ to $\mathbb{F}_{2}$ given by $\left(f \oplus F_{\ell}\right)\left(x_{i}\right)=f\left(x_{i}\right)+F_{\ell}\left(x_{i}\right)$.

We first show that $\varphi$ is a function to the vertices of $G_{\mathcal{F}_{0}}$. For a vertex $(\ell, f) \in V\left(G_{\mathcal{F}}\right)$, the constraint $C_{\ell}$ has the form $\sum_{x_{i} \in S_{\ell}} x_{i}=b_{\ell}$. By assumption, both $f$ and $F_{\ell}$ satisfy $C_{\ell}$, i.e., we have that $\sum_{x_{i} \in S_{\ell}} f\left(x_{i}\right)=b_{\ell}$ and $\sum_{x_{i} \in S_{\ell}} F_{\ell}\left(x_{i}\right)=b_{\ell}$. Adding these up we get $\sum_{x_{i} \in S_{\ell}}\left(f \oplus F_{\ell}\right)\left(x_{i}\right)=$ $b_{\ell}+b_{\ell}=0$ and so $\left(\ell, f \oplus F_{\ell}\right)$ is indeed a vertex of $G_{\mathcal{F}_{0}}$. It is also easy to see that $\varphi$ is an injection and therefore also a bijection.

Next we show that $\varphi$ preserves adjacency. Suppose that $(\ell, f)$ and $\left(k, f^{\prime}\right)$ are adjacent in $G_{\mathcal{F}}$. Then there exists $x_{i} \in S_{\ell} \cap S_{k}$ such that $f\left(x_{i}\right) \neq f^{\prime}\left(x_{i}\right)$. It is easy to see that $f\left(x_{i}\right)+F_{\ell}\left(x_{i}\right)=$ $f\left(x_{i}\right)+F\left(x_{i}\right) \neq f^{\prime}\left(x_{i}\right)+F\left(x_{i}\right)=f^{\prime}\left(x_{i}\right)+F_{k}\left(x_{i}\right)$ and thus $\left(\ell, f \oplus F_{\ell}\right)$ is adjacent to $\left(k, f^{\prime} \oplus F_{k}\right)$ in $G_{\mathcal{F}_{0}}$. So $\varphi$ preserves adjacency and the proof that it preserves non-adjacency is similar. This implies that $\varphi$ is an isomorphism and thus $G_{\mathcal{F}}$ and $G_{\mathcal{F}_{0}}$ are isomorphic.

$($ ii $) \Longrightarrow($ iii $)$. We have already seen in $(33)$ that for any linear BCS $\mathcal{F}$ with $m$ constraints we have that $\alpha\left(G_{\mathcal{F}_{0}}\right)=m$. By assumption $G_{\mathcal{F}} \cong G_{\mathcal{F}_{0}}$ and the claim follows. 
(iii) $\Longrightarrow(i)$. Finally, suppose that $\alpha\left(G_{\mathcal{F}}\right)=m$ and that $T$ is an independent set meeting this bound. As all vertices of $G_{\mathcal{F}}$ corresponding to a fixed constraint are pairwise adjacent, we must have that $T$ contains a unique vertex of the form $(\ell, f)$ for every $\ell \in[m]$. Therefore, we can define $f_{\ell}: S_{\ell} \rightarrow \mathbb{F}_{2}$ to be such that $\left(\ell, f_{\ell}\right) \in T$ for all $\ell \in[m]$. We will use these partial assignments to define a satisfying assignment $F$ for the BCS $\mathcal{F}$. Consider a variable $x_{i}$ and let $\ell \in[m]$ such that $x_{i} \in S_{\ell}$. We define $F\left(x_{i}\right)=f_{\ell}\left(x_{i}\right)$. It remains to show that $F$ is well-defined. Since $T$ is an independent set in $G_{\mathcal{F}}$, if $k \neq \ell$ and $x_{i} \in S_{k}$, we must have that $f_{\ell}\left(x_{i}\right)=f_{k}\left(x_{i}\right)$. Therefore, the restriction of $F$ to $S_{k}$ is equal to $f_{k}$ for all $k \in[m]$. This implies that $F$ satisfies all of the constraints and is therefore a satisfying assignment.

Next we prove the quantum analog of Theorem 6.2.

Theorem 6.3. Let $\mathcal{F}$ be a linear BCS with $m$ constraints. Then the following are equivalent:

(i) $\mathcal{F}$ is quantum satisfiable;

(ii) The graphs $G_{\mathcal{F}}$ and $G_{\mathcal{F}_{0}}$ are quantum isomorphic;

(iii) There exists a projective packing of $G_{\mathcal{F}}$ of value $m$;

(iv) $\alpha_{q}\left(G_{\mathcal{F}}\right)=m$.

Proof. $(i) \Longrightarrow($ ii $)$. Suppose that $\mathcal{F}$ is quantum satisfiable, i.e., there exists a perfect quantum strategy for the BCS game for $\mathcal{F}$. We now describe a perfect strategy for the $\left(G_{\mathcal{F}}, G_{\mathcal{F}_{0}}\right)$-isomorphism game that uses the perfect quantum strategy for the BCS game as a subroutine.

In the $\left(G_{\mathcal{F}}, G_{\mathcal{F}_{0}}\right)$-isomorphism game, Alice receives a vertex $\left(\ell_{A}, f_{A}\right) \in V\left(G_{\mathcal{F}}\right) \cup V\left(G_{\mathcal{F}_{0}}\right)$. Upon receiving her question, she uses the perfect strategy for the BCS game to obtain an assignment $f_{A}^{\prime}$ : $S_{\ell_{A}} \rightarrow \mathbb{F}_{2}$ that satisfies $C_{\ell_{A}}$ in $\mathcal{F}$, and responds with the vertex $\left(\ell_{A}, f_{A} \oplus f_{A}^{\prime}\right)$. Similarly, Bob receives a vertex $\left(\ell_{B}, f_{B}\right) \in V\left(G_{\mathcal{F}}\right) \cup V\left(G_{\mathcal{F}_{0}}\right)$. Using the perfect strategy for the BCS game he obtains an assignment $f_{B}^{\prime}$ satisfying $C_{\ell_{B}}$ and he responds with $\left(\ell_{B}, f_{B} \oplus f_{B}^{\prime}\right)$. Note that $\left(f_{A} \oplus f_{A}^{\prime}\right) \oplus f_{A}^{\prime}=f_{A}$ and so without loss of generality we may assume that $\left(\ell_{A}, f_{A}\right),\left(\ell_{B}, f_{B}\right) \in V\left(G_{\mathcal{F}}\right)$.

We now show that the strategy for the $\left(G_{\mathcal{F}}, G_{\mathcal{F}_{0}}\right)$-isomorphism game described above is perfect. For this, we need to show that Conditions (1) and (2) are satisfied. First, note that since $f_{A}^{\prime}$ satisfies constraint $C_{\ell_{A}}$ in $\mathcal{F}$, Alice's output $\left(\ell_{A}, f_{A} \oplus f_{A}^{\prime}\right)$ is in $V\left(G_{\mathcal{F}}\right)$ if her input $\left(\ell_{A}, f_{A}\right)$ was in $V\left(G_{\mathcal{F}_{0}}\right)$ and vice versa, thus Condition (1) of the isomorphism game is met. Second, suppose that Alice and Bob's inputs were equal. Since they are using a perfect strategy for the BCS game, the functions $f_{A}^{\prime}$ and $f_{B}^{\prime}$ are also the same and thus their outputs $\left(\ell_{A}, f_{A} \oplus f_{A}^{\prime}\right)$ and $\left(\ell_{B}, f_{B} \oplus f_{B}^{\prime}\right)$ are equal. Third, suppose that their inputs $\left(\ell_{A}, f_{A}\right)$ and $\left(\ell_{B}, f_{B}\right)$ were adjacent. By definition, there exists $x_{i} \in S_{\ell_{A}} \cap S_{\ell_{B}}$ such that $f_{A}\left(x_{i}\right) \neq f_{B}\left(x_{i}\right)$. However, since they are using a perfect strategy for the BCS game, we have that $f_{A}^{\prime}\left(x_{i}\right)=f_{B}^{\prime}\left(x_{i}\right)$ and thus $\left(f_{A} \oplus f_{A}^{\prime}\right)\left(x_{i}\right) \neq\left(f_{B} \oplus f_{B}^{\prime}\right)\left(x_{i}\right)$. Therefore, their outputs $\left(\ell_{A}, f_{A} \oplus f_{A}^{\prime}\right)$ and $\left(\ell_{B}, f_{B} \oplus f_{B}^{\prime}\right)$ will be adjacent. Lastly, the proof that they output distinct non-adjacent vertices upon receiving distinct non-adjacent input vertices is similar. Therefore, Alice and Bob can win the $\left(G_{\mathcal{F}}, G_{\mathcal{F}_{0}}\right)$-isomorphism game perfectly with this strategy. Since the strategy they used for the BCS game could be realized by quantum measurements on a shared entangled state, so can this one. Thus $G_{\mathcal{F}} \cong_{q} G_{\mathcal{F}_{0}}$ and the proof is concluded.

$($ ii $) \Longrightarrow\left(\right.$ iii). Suppose that $G_{\mathcal{F}}$ and $G_{\mathcal{F}_{0}}$ are quantum isomorphic. By (33) we have that $\alpha\left(G_{\mathcal{F}_{0}}\right)=m$. By Remark 5.17, it follows that $G_{\mathcal{F}_{0}}$ has a projective packing of value $m$. Since $G_{\mathcal{F}} \cong_{q} G_{\mathcal{F}_{0}}$, it follows by Lemma 5.18 that $G_{\mathcal{F}}$ must also have a projective packing of value $m$. 
$($ iii $) \Longrightarrow(i)$. Suppose that $G_{\mathcal{F}}$ has a projective packing $(\ell, f) \mapsto E_{(\ell, f)} \in \mathbb{C}^{d \times d}$ of value $m$. Since the vertices corresponding to a single constraint form a clique, we have that the projectors assigned to those vertices are all mutually orthogonal. From this it follows that

$$
\sum_{f:(\ell, f) \in V\left(G_{\mathcal{F}}\right)} \operatorname{rk}\left(E_{(\ell, f)}\right) \leq d, \text { for all } \ell \in[m] .
$$

Furthermore, we have that

$$
m=\frac{1}{d} \sum_{(\ell, f) \in V\left(G_{\mathcal{F}}\right)} \operatorname{rk}\left(E_{(\ell, f)}\right)=\frac{1}{d} \sum_{\ell \in[m]} \sum_{f:(\ell, f) \in V\left(G_{\mathcal{F}}\right)} \operatorname{rk}\left(E_{(\ell, f)}\right) \leq \frac{1}{d} m d=m,
$$

where for the inequality we used (34). Thus, equality holds throughout in (35). This implies that

$$
\sum_{f:(\ell, f) \in V\left(G_{\mathcal{F}}\right)} \operatorname{rk}\left(E_{(\ell, f)}\right)=d, \text { for all } \ell \in[m],
$$

which is possible if and only if

$$
\sum_{f:(\ell, f) \in V\left(G_{\mathcal{F}}\right)} E_{(\ell, f)}=I_{d}
$$

In view of (36), the matrices $\left\{E_{(\ell, f)}: f\right.$ satisfies $\left.C_{\ell}\right\}$ form quantum measurement for each $\ell \in[m]$. To conclude the proof, we use these measurements to construct a perfect quantum strategy for the BCS game for $\mathcal{F}$. Specifically, the players share the maximally entangled state $\psi_{d}=$ $\frac{1}{\sqrt{d}} \sum_{i=1}^{d} e_{i} \otimes e_{i}$. Upon receiving constraint $C_{\ell}$, Alice performs the measurement $\left\{E_{(\ell, f)}: f\right.$ satisfies $\left.C_{\ell}\right\}$ on her half of $\psi_{d}$ to obtain an assignment $f: S_{\ell} \rightarrow \mathbb{F}_{2}$ that satisfies $C_{\ell}$. Upon receiving constraint $C_{k}$, Bob acts similarly, except that he performs the measurement $\left\{E_{(k, f)}^{\top}: f\right.$ satisfies $\left.C_{k}\right\}$ to get an assignment $f^{\prime}: S_{k} \rightarrow \mathbb{F}_{2}$ that satisfies $C_{k}$. The corresponding correlation is given by

$$
p\left(f, f^{\prime} \mid C_{\ell}, C_{k}\right)=\psi_{d}^{\dagger}\left(E_{(\ell, f)} \otimes E_{\left(k, f^{\prime}\right)}^{\top}\right) \psi_{d}=\frac{1}{d} \operatorname{Tr}\left(E_{(\ell, f)} E_{\left(k, f^{\prime}\right)}\right),
$$

where for the last equality we use (3).

It remains to check that this strategy wins the BCS game for $\mathcal{F}$ perfectly. For this we need to show that the correlation defined in (37) evaluates to zero whenever the winning conditions of the BCS game are not fulfilled (recall Equation (4)). Now, by construction of the measurements, Alice and Bob always output an assignment that satisfies their individual constraints. So the only thing to check is that the players are consistent on any variables contained in both of their constraints. However, if there exists $x_{i} \in S_{\ell} \cap S_{k}$ such that $f\left(x_{i}\right) \neq f^{\prime}\left(x_{i}\right)$, then the vertices $(\ell, f)$ and $\left(k, f^{\prime}\right)$ are adjacent in $G_{\mathcal{F}}$. Therefore, the projectors $E_{(\ell, f)}$ and $E_{\left(k, f^{\prime}\right)}$ are orthogonal since they originated from a projective packing. As a consequence, it follows by (37) that the probability of Alice and Bob responding with $f$ and $f^{\prime}$ respectively upon being given constraints $C_{\ell}$ and $C_{k}$ is equal to zero.

$($ iii $) \Longrightarrow(i v)$. First, note that we can color the complement of $G_{\mathcal{F}}$ with $m$ colors because the vertices corresponding to a fixed constraint of $\mathcal{F}$ are an independent set in $\overline{G_{\mathcal{F}}}$. Therefore, $\chi\left(\overline{G_{\mathcal{F}}}\right) \leq m$. Thus if there exists a projective packing of value $m$, by Lemma 5.19 we have that $\chi\left(\overline{G_{\mathcal{F}}}\right)=m$ and that $\alpha_{q}\left(G_{\mathcal{F}}\right)=m$. 
$(i v) \Longrightarrow(i i i)$. Conversely, if $\alpha_{q}\left(G_{\mathcal{F}}\right)=m$ then we must have that $\chi\left(\overline{G_{\mathcal{F}}}\right)=m$. Then Lemma 5.19 implies that there exists a projective packing of value $m$.

As a corollary of the above two theorems, we have that isomorphism and quantum isomorphism are distinct relations on graphs:

Theorem 6.4. There exists graphs that are quantum isomorphic but not isomorphic. In particular, if $\mathcal{F}$ is a linear BCS that is quantum satisfiable but not satisfiable, then the graphs $G_{\mathcal{F}}$ and $G_{\mathcal{F}_{0}}$ are quantum isomorphic but not isomorphic.

The smallest example of a quantum satisfiable but not satisfiable linear BCS that we know of is the Mermin magic square BCS given in (32). The two corresponding graphs each have 24 vertices. Interestingly, both of the graphs have automorphism groups that act transitively on their vertices. In fact, both of the graphs are Cayley graphs. We present the two graphs in Figures 1 and 2 below.

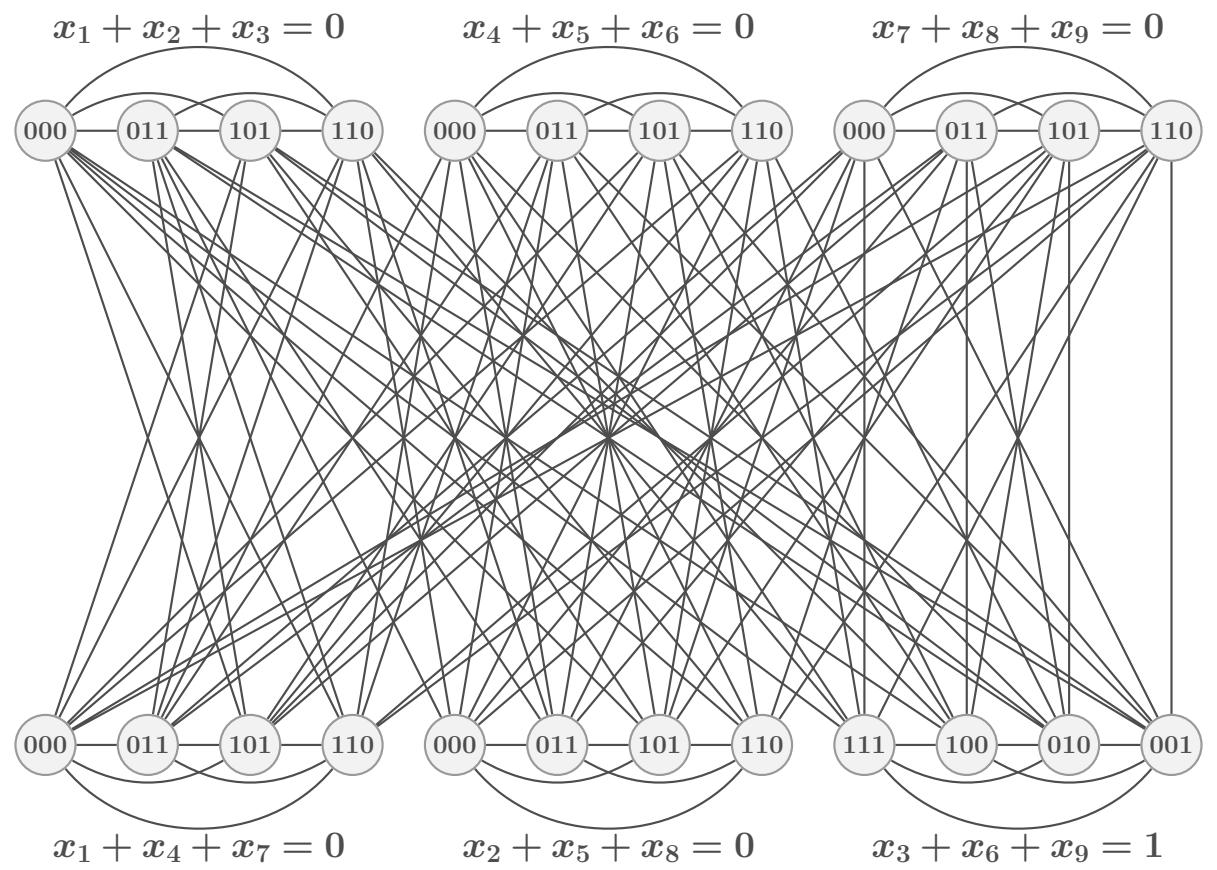

Figure 1: $G(\mathcal{F})$ for the Mermin magic square game $(32)$.

We note here that the first separating example that we constructed was slightly different than the one presented above. It was a version of the celebrated CFI construction, named after Cai, Fürer, and Immerman [8]. The original CFI construction was designed to produce pairs of non-isomorphic graphs that cannot be distinguished by the $d$-dimensional Weisfeiler-Lehman algorithm for any fixed $d$. The CFI construction was reinterpreted by Atserias, Bulatov, and Dawar [2] to view it as an encoding of special systems of linear equations over $\mathbb{Z}_{2}$, where each variable appears in precisely two equations. Our first separating example was literally the CFI construction corresponding to a system of linear equations as in [2], in which each variable appears in exactly two equations, and that is classically unsatisfiable over $\mathbb{Z}_{2}$ but quantum satisfiable. The Mermin-Peres magic square game gives rise to such a system of linear equations. When applied to the constraint system describing 


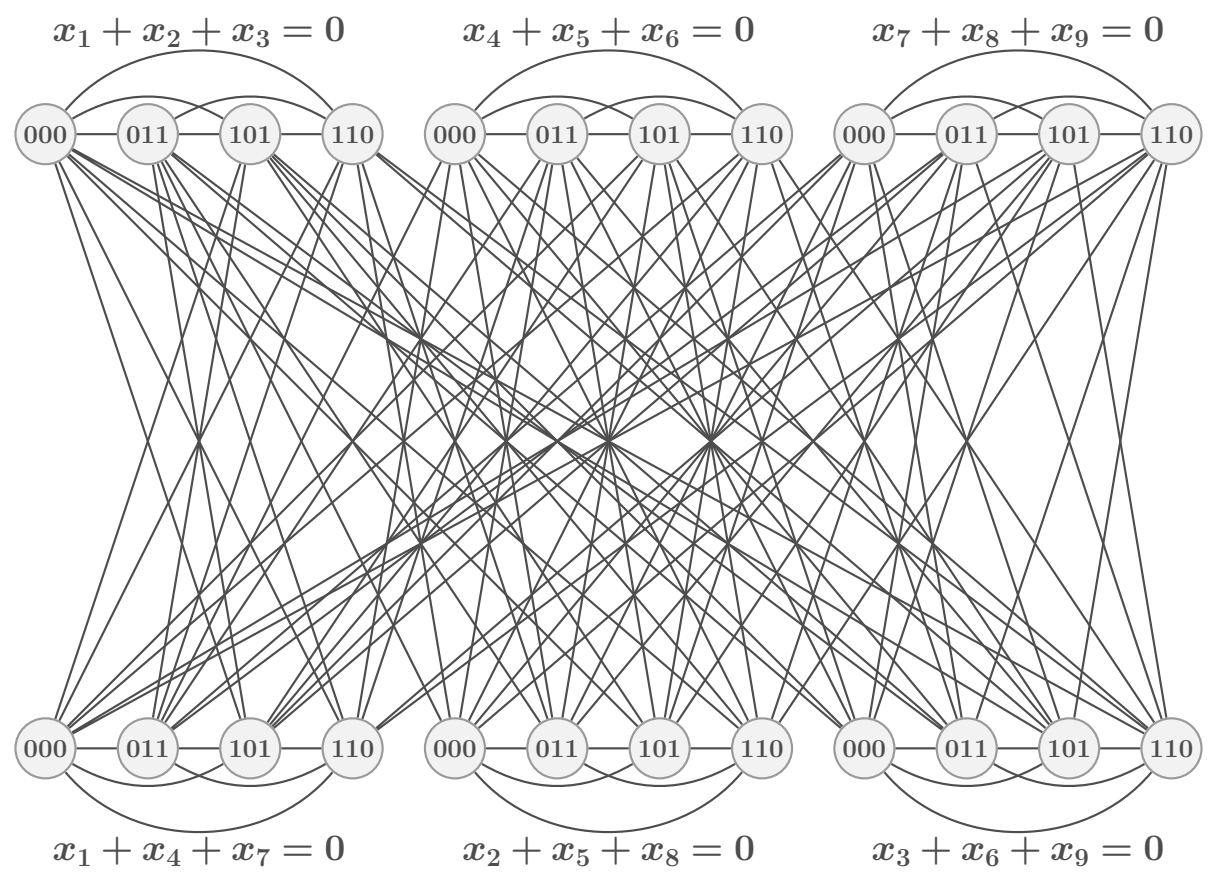

Figure 2: $G\left(\mathcal{F}_{0}\right)$ for the Mermin magic square game 32 .

the magic square, this construction produced graphs with several hundred vertices. The final construction which we described above is a simplified version of this, in which several vertices have been merged together, and several others have been removed, without changing the outcome. The final graphs have a few dozen vertices. As it turns out, this streamlined version of the construction is quite similar to the FGLSS reduction from the theory of hardness of approximation [12], which interpreted in this context is a reduction from the feasibility problem for arbitrary systems of linear equations over $\mathbb{Z}_{2}$ (without any restriction on the number of occurrences of each variable) to the graph isomorphism problem. The FGLSS construction was also used in the context of the graph isomorphism problem in [23].

In [32, Slofstra showed that determining the existence of a perfect quantum strategy for linear BCS game is undecidable. Combining this with our reduction, we obtain the following:

Corollary 6.5. It is undecidable to determine if two graphs are quantum isomorphic.

\section{Separating quantum and quantum commuting isomorphism}

We can also apply the techniques of the previous section to show that the existence of quantum commuting strategies for a linear BCS game can be reduced to the existence of a quantum commuting strategy for a corresponding isomorphism game. We first introduce some definitions.

For our results on quantum commuting isomorphism, we will need an analog of projective packings that allows the projections we assign to our vertices to be more general objects. Such an analog of projective representations/projective rank was given in [25], and we can adapt their approach here. Let $\mathcal{A}$ be a unital $C^{*}$-algebra that admits a faithful tracial state $s$. An assignment, 
$g \mapsto E_{g} \in \mathcal{A}$, of projections from $\mathcal{A}$ to the vertices of a graph $G$ is a tracial packing of $G$ if $E_{g} E_{g^{\prime}}=0$ whenever $g \sim g^{\prime}$. The value of a such a tracial packing is $\sum_{g \in V(G)} s\left(E_{g}\right)=s\left(\sum_{g \in V(G)} E_{g}\right)$. The tracial packing number of $G$, denoted $\alpha_{t r}(G)$, is the supremum of values of tracial packings of $G$.

We also need to introduce quantum commuting homomorphisms and the quantum commuting independence number of a graph $G$, denoted $\alpha_{q c}(G)$, analogously to quantum homomorphisms. We note that quantum commuting homomorphisms have been investigated in [25] and [24].

We are now ready to give an analog of Theorem 6.3 for quantum commuting strategies:

Theorem 7.1. Let $\mathcal{F}$ be a linear BCS with $m$ constraints. Then the following are equivalent:

(i) The BCS game for $\mathcal{F}$ has a perfect quantum commuting strategy;

(ii) The graphs $G_{\mathcal{F}}$ and $G_{\mathcal{F}_{0}}$ are quantum commuting isomorphic;

(iii) There exists a tracial packing of $G_{\mathcal{F}}$ of value $m$;

(iv) $\alpha_{q c}\left(G_{\mathcal{F}}\right)=m$.

Proof. The proof follows the same format as that of Theorem 6.3. Indeed, note that proof $(i) \Rightarrow(i i)$ from above is independent of the type of strategy used, i.e., it works just as well for the quantum commuting case. The proof of $(i i) \Rightarrow(i i i)$ is identical as well, with tracial packing replacing projective packing. To show that $(i i i) \Rightarrow(i)$, the main difference is to just use $s\left(E_{(\ell, f)}\right)$ in place of $\operatorname{rk}\left(E_{(\ell, f)}\right) / d$, and the fact that for a faithful tracial state $s$, the equality $\sum_{i \in[r]} s\left(E_{i}\right)=1$ implies that $\sum_{i \in[r]} E_{i}=I$ for any mutually orthogonal projectors $E_{1}, \ldots, E_{r}$. It is then easy to see that the projectors $E_{(\ell, f)}$ satisfy Lemma 5.13 and thus the BCS game for $\mathcal{F}$ has a perfect quantum commuting strategy. The equivalence of $(i i i)$ and $(i v)$ is identical to the above, just using the tracial packing analog of Lemma 5.19 .

For our main results on quantum commuting isomorphism, we will use the above along with the following two results of Slofstra [31]:

Theorem 7.2 (Slofstra). There is a linear BCS game that has a perfect quantum commuting strategy but no perfect quantum strategy.

Theorem 7.3 (Slofstra). It is undecidable to determine if a linear BCS game has a perfect quantum commuting strategy.

From our Theorem 7.1 and the above two theorems of Slofstra we immediately obtain the desired corollaries:

Corollary 7.4. There exist graphs $G$ and $H$ such that $G \cong_{q c} H$ but $G \varlimsup_{q} H$.

Corollary 7.5. It is undecidable to determine if two graphs are quantum commuting isomorphic.

Unfortunately, the linear binary constraint systems that Slofstra uses to prove Theorem 7.2 are too large to produce graphs of reasonable size, and thus we cannot include any specific examples for Corollary 7.4. Recall that Theorem 4.5 states that non-signalling isomorphism and fractional isomorphism are equivalent relations, and further recall that the latter is known to be polynomial-time decidable. Thus Corollary 7.5 implies that quantum commuting isomorphism and non-signalling isomorphism are distinct relations:

Theorem 7.6. There exist graphs $G$ and $H$ such that $G \cong_{n s} H$ but $G \varlimsup_{q c} H$. 
Combining the results of this section with Theorem 6.4, we see that isomorphism, quantum isomorphism, quantum commuting isomorphism, and non-signalling isomorphism are all distinct relations, i.e., none of the implications in Equation (31) can be reversed. Furthermore, the variation in complexity among these four relations is quite interesting. The weakest among them, nonsignalling isomorphism, is polynomial-time decidable, but the next two strongest, quantum and quantum commuting isomorphism, are undecidable. Of course isomorphism itself was recently shown by Babai [5] to be decidable in quasipolynomial time.

\section{Acknowledgements}

AA was partially funded by the European Research Council (ERC) under the European Union's Horizon 2020 research and innovation programme, grant agreement ERC-2014-CoG 648276 (AUTAR), and by MINECO through TIN2013-48031-C4-1-P (TASSAT2). LM is supported by UK EPSRC under grant EP/L021005/1. DR is supported by Cambridge Quantum Computing Ltd. and the Engineering and Physical Sciences Research Council of the United Kingdom (EPSRC), as well as Simone Severini and Fernando Brandao. RS is partially supported by grant GA ČR P202-12G061 and by grant LL1201 ERC CZ of the Czech Ministry of Education, Youth and Sports. SS is supported by the Royal Society, the EPSRC, and the National Natural Science Foundation of China (NSFC). AV is supported in part by the Singapore National Research Foundation under NRF RF Award No. NRF-NRFF2013-13. Part of this work was done while AA, DR, and SS were visiting the Simons Institute for the Theory of Computing.

\section{References}

[1] Alex Arkhipov. Extending and characterizing quantum magic games. 2012. arXiv:1209.3819.

[2] Albert Atserias, Andrei Bulatov, and Anuj Dawar. Affine systems of equations and counting infinitary logic. Theoretical Computer Science, 410(18):1666 - 1683, 2009. doi:10.1016/j. tcs.2008.12.049.

[3] Albert Atserias and Elitza N. Maneva. Sherali-Adams Relaxations and Indistinguishability in Counting Logics. SIAM J. Comput., 42(1):112-137, 2013. doi:10.1137/120867834.

[4] László Babai. Automorphism groups, isomorphism, reconstruction. In Handbook of Combinatorics (Vol. 2), pages 1447-1540. 1995.

[5] László Babai. Graph isomorphism in quasipolynomial time. In Proceedings of the fortyeighth annual ACM symposium on Theory of Computing, pages 684-697, 2016. doi:10.1145/ 2897518.2897542.

[6] László Babai, Paul Erdős, and Stanley M. Selkow. Random graph isomorphism. SIAM Journal on Computing, 9(3):628-635, 1980. doi:10.1137/0209047.

[7] Christoph Berkholz and Martin Grohe. Linear Diophantine equations, group CSPs, and graph isomorphism. In Proceedings of the Twenty-Eighth Annual ACM-SIAM Symposium on Discrete Algorithms, pages 327-339, 2017. 
[8] Jin-Yi Cai, Martin Fürer, and Neil Immerman. An optimal lower bound on the number of variables for graph identification. Combinatorica, 12(4):389-410, 1992. doi:10.1007/BF01305232

[9] Peter J. Cameron, Ashley Montanaro, Michael W. Newman, Simone Severini, and Andreas Winter. On the quantum chromatic number of a graph. Electronic Journal of Combinatorics, 14(1), 2007. arXiv:quant-ph/0608016.

[10] Richard Cleve, Li Liu, and William Slofstra. Perfect commuting-operator strategies for linear system games. Journal of Mathematical Physics, 58(1), 2017. doi:10.1063/1.4973422.

[11] Richard Cleve and Rajat Mittal. Characterization of binary constraint system games. In Proceedings of the 41 st International Colloquium on Automata, Languages, and Programming, ICALP '14, pages 320-331. 2014. arXiv:1209.2729.

[12] Uriel Feige, Shafi Goldwasser, Laszlo Lovász, Shmuel Safra, and Mario Szegedy. Interactive proofs and the hardness of approximating cliques. Journal of the ACM, 43(2):268-292, March 1996. doi:10.1145/226643.226652.

[13] Martin Grohe. Descriptive complexity, canonisation, and definable graph structure theory, volume 47. Cambridge University Press, 2017. doi:10.1017/9781139028868.

[14] Martin Grohe and Martin Otto. Pebble games and linear equations. Journal of Symbolic Logic, 80(03), 2012. doi:10.1017/jsl.2015.28.

[15] Laura Mancinska, David E. Roberson, Robert Samal, Simone Severini, and Antonios Varvitsiotis. Relaxations of Graph Isomorphism. In 44th International Colloquium on Automata, Languages, and Programming (ICALP 2017), volume 80 of Leibniz International Proceedings in Informatics (LIPICs), pages 76:1-76:14, 2017. doi:10.4230/LIPIcs. ICALP.2017.76.

[16] Laura Mančinska and David E. Roberson. Quantum homomorphisms. Journal of Combinatorial Theory, Series B, 118:228 - 267, 2016. doi:10.1016/j.jctb.2015.12.009.

[17] Laura Mančinska, David E. Roberson, and Antonios Varvisotis. On deciding the existence of perfect entangled strategies for nonlocal games. Chicago Journal of Theoretical Computer Science, 2016(5), 2016. doi:10.4086/cjtcs.2016.005.

[18] Laura Mančinska, David E. Roberson, and Antonios Varvitsiotis. Semidefinite relaxations of quantum isomorphism. Available online, 2016.

[19] Brendan D. McKay and Adolfo Piperno. Practical graph isomorphism, II. Journal of Symbolic Computation, 60:94 - 112, 2014. doi:10.1016/j.jsc.2013.09.003.

[20] Nathaniel D. Mermin. Simple unified form for the major no-hidden-variables theorems. Physical Review Letters, 65:3373-3376, 1990. doi:10.1103/PhysRevLett.65.3373.

[21] Benjamin Musto and Jamie Vicary. Quantum latin squares and unitary error bases. Quantum Information \& Computation, 16(15-16):1318-1332, November 2016. URL: http://dl.acm. org/citation. $\mathrm{cfm}$ ?id=3179439.3179443.

[22] Michael A. Nielsen and Isaac L. Chuang. Quantum Computation and Quantum Information. Cambridge University Press, 2010. 
[23] Ryan O'Donnell, John Wright, Chenggang Wu, and Yuan Zhou. Hardness of Robust Graph Isomorphism, Lasserre Gaps, and Asymmetry of Random Graphs, pages 1659-1677. 2014. arXiv:1401.2436.

[24] Carlos M. Ortiz and Vern I. Paulsen. Quantum graph homomorphisms via operator systems. Linear Algebra and its Applications, 497:23 - 43, 2016. doi:10.1016/j.laa.2016.02.019.

[25] Vern I. Paulsen, Simone Severini, Dan Stahlke, Ivan G. Todorov, and Andreas Winter. Estimating quantum chromatic numbers. Journal of Functional Analysis, 270(6):2188-2222, 2016. doi:10.1016/j.jfa.2016.01.010.

[26] Vern I. Paulsen and Ivan G. Todorov. Quantum chromatic numbers via operator systems. The Quarterly Journal of Mathematics, 66(2):677-692, 2015. doi:10.1093/qmath/hav004.

[27] Sandu Popescu and Daniel Rohrlich. Quantum nonlocality as an axiom. Foundations of Physics, 24(3):379-385, 1994. doi:10.1007/BF02058098.

[28] Motakuri V. Ramana, Edward R. Scheinerman, and Daniel Ullman. Fractional isomorphism of graphs. Discrete Mathematics, 132(1):247 - 265, 1994. doi:10.1016/0012-365X (94) 90241-0.

[29] David E. Roberson. Variations on a Theme: Graph Homomorphisms. PhD thesis, University of Waterloo, 2013.

[30] Volkher B. Scholz and Reinhard F. Werner. Tsirelson's problem. 2008. arXiv:0812.4305.

[31] William Slofstra. Tsirelson's problem and an embedding theorem for groups arising from non-local games. 2016. arXiv:1606.03140.

[32] William Slofstra. The set of quantum correlations is not closed. 2017. arXiv:1703.08618.

[33] Gottfried Tinhofer. Graph isomorphism and theorems of Birkhoff type. Computing, 36(4):285300, 1986. doi:10.1007/BF02240204.

[34] Boris Tsirelson. Bell inequalities and operator algebras. Problem 33, 6 July 2006, Open Problems in Quantum Information Theory, Institut für Mathematische Physik, TU Braunschweig, Germany. URL: https://www.tau.ac.il/ tsirel/download/bellopalg.pdf.

[35] Edwin R. van Dam and Willem H. Haemers. Which graphs are determined by their spectrum? Linear Algebra and its Applications, 373:241 -272, 2003. doi:10.1016/S0024-3795(03) 00483-X. 